\title{
INTERPOLATION OF NON-SMOOTH FUNCTIONS ON ANISOTROPIC FINITE ELEMENT MESHES
}

\author{
Thomas ApeL ${ }^{1}$
}

\begin{abstract}
In this paper, several modifications of the quasi-interpolation operator of Scott and Zhang [30] are discussed. The modified operators are defined for non-smooth functions and are suited for application on anisotropic meshes. The anisotropy of the elements is reflected in the local stability and approximation error estimates. As an application, an example is considered where anisotropic finite element meshes are appropriate, namely the Poisson problem in domains with edges.
\end{abstract}

AMS Subject Classification. 65D05, 65N30, 65N50.

Received: October 9, 1997. Revised: April 3, 1998 and March 26, 1999.

\section{INTRODUCTION}

The solution of elliptic boundary value problems may have anisotropic behaviour near certain manifolds $M \subset \bar{\Omega}$. That means that the solution varies significantly only perpendicularly to $M$. Examples include the Poisson problem in domains with edges $M$ and singularly perturbed convection diffusion reaction problems where $M$ is part of the boundary or an internal manifold. In such cases it is an obvious idea to reflect this anisotropy in the discretization by using anisotropic meshes with a small mesh size in the direction of the rapid variation of the solution and a larger mesh size in the perpendicular direction.

In order to describe the elements of anisotropic meshes mathematically, consider an elliptic boundary value problem posed over a polyhedral domain $\Omega \subset \mathbb{R}^{d}, d=2,3$. We study the discretization error of the finite element method on a family of meshes $\mathcal{T}_{h}=\{e\}$ with the usual admissibility conditions (see, for example, Conditions $\left(\mathcal{T}_{h} 1-\mathcal{T}_{h} 5\right)$ in Chapter 2 of [18]). Denote by $h_{e}$ the diameter of the finite element $e$, and by $\varrho_{e}$ the supremum of the diameters of all balls contained in $e$. Then it is assumed in the classical finite element theory that $h_{e} \lesssim \varrho_{e}$, for the definition of $\lesssim$ see Section 2 . This assumption is no longer valid in the case of anisotropic meshes. Conversely, anisotropic elements $e$ are characterized by

$$
\frac{h_{e}}{\varrho_{e}} \rightarrow \infty
$$

where the limit can be considered as $h \rightarrow 0$ (see the application to the Poisson equation in [4,9] or Section 7) or $\varepsilon \rightarrow 0$ where $\varepsilon$ is some (small perturbation) parameter of the problem (see the singularly perturbed problems in $[6,7])$.

Keywords and phrases. Anisotropic finite elements, interpolation error estimate, quasi-interpolation, non-smooth functions, edge singularity, reaction diffusion problem.

1 TU Chemnitz, Fak. f. Mathematik, 09107 Chemnitz, Germany. e-mail: apel@mathematik.tu-chemnitz.de 

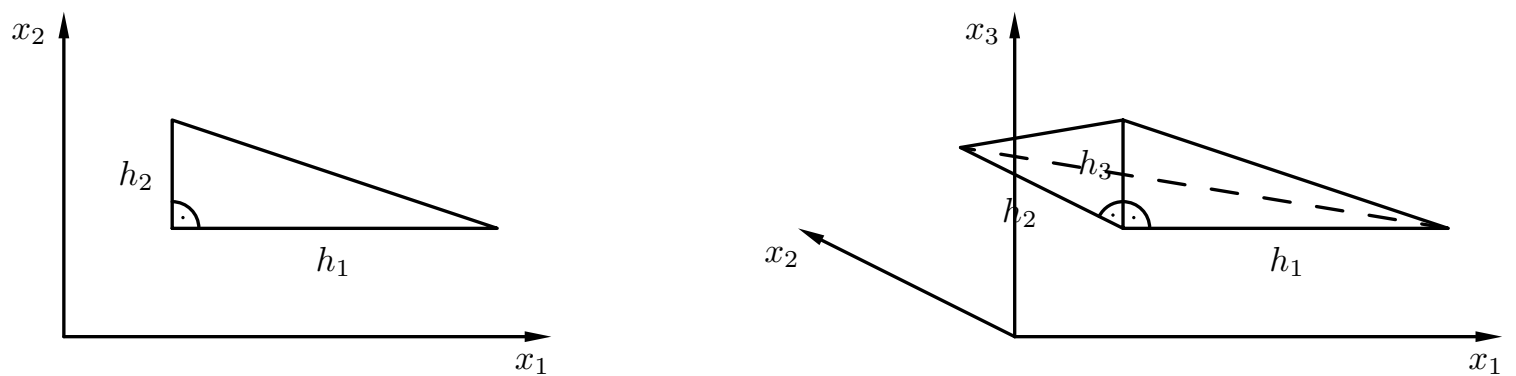

FIGURE 1. Illustration of the simplest anisotropic finite elements.

Local estimates of the interpolation error are basic ingredients for a priori estimates of the finite element error, for proving the equivalence of error estimators and the exact error, and for investigating multi-level algorithms for the solution of the system of algebraic equations which arise in the finite element method. For Lagrangian finite elements, the simplest approximation is the nodal interpolant $\mathrm{I}_{h}: C(\bar{\Omega}) \rightarrow V_{h}:=\operatorname{span}\left\{\varphi_{i}, i \in I\right\}$,

$$
\left(\mathrm{I}_{h} u\right)(x):=\sum_{i \in I} u\left(X_{i}\right) \varphi_{i}(x)
$$

where $X_{i}$ are the nodes and $\varphi_{i}(x)$ are the nodal basis functions, $\varphi_{i}\left(X_{j}\right)=\delta_{i j}$ for all $i, j \in I$. Because $\mathrm{I}_{h}$ is defined locally on every element the interpolation error $u-\mathrm{I}_{h} u$ can be estimated elementwise. Before we discuss the drawback of the nodal interpolant we shall recall some anisotropic interpolation error estimates. We denote error estimates as anisotropic if they are sharp enough to reflect the different element sizes and not only the diameter.

For simplicity in this Introduction consider a triangle or a tetrahedron $e \subset \mathbb{R}^{d}$ with element sizes $h_{1}, \ldots, h_{d}$ as given in Figure 1. That means that the element $e$ has $d$ edges of length $h_{i}$ which are parallel to the corresponding coordinate axes. Then for linear elements the following estimates hold $[4,7]$ :

$$
\begin{gathered}
\left\|u-\mathrm{I}_{h} u ; L^{p}(e)\right\| \lesssim \sum_{|\alpha|=\ell} h^{\alpha}\left\|D^{\alpha} u ; L^{p}(e)\right\|, \quad \text { if }\left\{\begin{array}{r}
\ell=1 \text { and } p \in(d, \infty], \\
\ell=2 \text { and } p \in[1, \infty],
\end{array}\right. \\
\left|u-\mathrm{I}_{h} u ; W^{1, p}(e)\right| \lesssim \sum_{|\alpha|=1} h^{\alpha}\left|D^{\alpha} u ; W^{1, p}(e)\right|, \quad \text { if } d=2 \text { or } p \in(2, \infty] .
\end{gathered}
$$

For the notation see Section 2. The necessity of the condition $p>2$ in the three-dimensional case is discussed at several places [4,22,31]. In the sequel, we will call an estimate to be of type $(m, n)$ if certain $m$ th derivatives (left-hand side) are estimated against $n$th derivatives of the solution. In this sense estimate (1.3) is of type (1,2).

For some applications, the nodal interpolant is not appropriate. First, the main drawback is that nodal values of $u$ have to be well-defined for the definition of $\mathrm{I}_{h} u$. For example, the solution of the Poisson equation with mixed boundary conditions can be of such poor regularity in the neighbourhood of edges that $u \notin W^{s, 2}(\Omega)$ for any $s>3 / 2$. This causes the interpolation theory with $\mathrm{I}_{h}$ to fail. Second, estimate (1.3) holds only for $p>2$ in the three-dimensional case. But $p=2$ is the natural choice in the investigation of the finite element approximation error. Using $p>2$ and the Hölder inequality leads to sub-optimal results, see the discussion in Section 7 . Third, there is no estimate of type $(1,1)$ for the nodal interpolant. Such estimates are of advantage for the investigation of multi-grid/multi-level methods for the solution of the system of algebraic equations which arise in the finite element method.

As a remedy, other approximation operators $\mathrm{Q}_{h}$ with $\mathrm{Q}_{h} u \in V_{h}$ can be considered. They are sometimes called quasi-interpolants and should preserve the following favourable properties of $\mathrm{I}_{h}$. 
1. $\mathrm{Q}_{h} u$ shall be defined locally. This means, that $\left(\mathrm{Q}_{h} u\right)(x)$ with $x \in e$ shall depend only on the values of $u$ in a small neighbourhood $S_{e}$ of $e$, where $S_{e}$ consists of a finite number (independent of $h$ ) of elements of $\mathcal{T}_{h}$. (For the nodal interpolant $\mathrm{I}_{h}$ we had in particular $S_{e}=e$.)

2. If possible, $\mathrm{Q}_{h}$ shall reproduce piecewise polynomials: $\mathrm{Q}_{h} u_{h}=u_{h}$ for all $u_{h} \in V_{h}$.

For isotropic meshes such operators have been studied in the literature. For an introduction, define by a generalization of (1.1)

$$
\left(\mathrm{Q}_{h} u\right)(x):=\sum_{i \in I} a_{i} \varphi_{i}(x)
$$

with real numbers $a_{i}$ still to be specified. Note that $\mathrm{Q}_{h}=\mathrm{I}_{h}$ if $a_{i}=u\left(X_{i}\right)$ for all $i \in I$.

In order to treat non-smooth functions the idea is to consider subdomains $\sigma_{i} \subset \bar{\Omega}$ (their choice will be discussed later), to define an $L^{2}$-projection operator

$$
\Pi_{\sigma_{i}}: L^{2}\left(\sigma_{i}\right) \rightarrow \mathcal{P}_{k, \sigma_{i}}
$$

and to choose

$$
a_{i}:=\left(\Pi_{\sigma_{i}} u\right)\left(X_{i}\right),
$$

for the notation see Section 2, for more details see (3.1-3.3). The numbers $a_{i}$ can be considered as averaged values of $u$ in $X_{i}$. Different authors chose different $\sigma_{i}$ resulting in different quasi-interpolation operators. We will now introduce three of them. For unambiguous reference we distinguish them by different symbols, $\mathrm{C}_{h}$, $\mathrm{O}_{h}$, and $\mathrm{Z}_{h}$.

Clément [19] uses $\overline{\sigma_{i}}:=\bigcup_{\bar{e} \ni X_{i}} \bar{e}$. The resulting operator $\mathrm{C}_{h}$,

$$
\left(\mathrm{C}_{h} u\right)(x):=\sum_{i \in I}\left(\Pi_{\sigma_{i}} u\right)\left(X_{i}\right) \cdot \varphi_{i}(x)
$$

is even defined for $u \in L^{1}(\Omega)$ and allows estimates of type $(m, \ell)$ for all $0 \leq m \leq \ell \leq k+1, k \geq 1$ is defined in Section 2. However, the operator $\mathrm{C}_{h}$ in this original form does not satisfy Property 2 above, but this can be corrected by defining

$$
\Pi_{\sigma_{i}}:\left.L^{2}\left(\sigma_{i}\right) \rightarrow V_{h}\right|_{\sigma_{i}}
$$

A modification of the Clément operator is discussed by Oswald [28]. For defining $\sigma_{i}$, he fixes just one (arbitrary) element $e=: \sigma_{i}$ with $X_{i} \in \bar{e}$. The resulting operator $\mathrm{O}_{h}$ allows the same estimates as $\mathrm{C}_{h}$, but we have $\left.V_{h}\right|_{\sigma_{i}}=\mathcal{P}_{k, \sigma_{i}}$. Some more details on $\mathrm{C}_{h}$ and $\mathrm{O}_{h}$ are given at the end of Section 3 when more notation has been introduced and more ideas have been developed.

The disadvantage of both $\mathrm{C}_{h}$ and $\mathrm{O}_{h}$ is that they do not preserve Dirichlet boundary conditions. For this reason, Scott and Zhang [30] modified again the choice of $\sigma_{i}$ and used not only $d$-dimensional subdomains $\sigma_{i}$ but also $(d-1)$-dimensional ones. In particular, they chose $\sigma_{i} \subset \partial \Omega$ if $X_{i} \in \partial \Omega$. Because we exploit this idea in this paper we will introduce the resulting operator $Z_{h}$ in more detail in Section 3. In particular, we derive some anisotropic estimates of type $(0, \ell), 1 \leq \ell \leq k+1$, and show that the operator $\mathrm{Z}_{h}$ has to be modified for error estimates of type $(1, \ell)$.

The aim of the paper is to define and to investigate quasi-interpolation operators which do not have the disadvantages of the Lagrange interpolation operator (see above) and which allow for proving anisotropic estimates of type $(m, \ell)$, with $m \geq 0$, for anisotropic meshes. Using the idea of lower-dimensional subdomains $\sigma_{i}$ we define in Sections 4-6 three operators of that type, $\mathrm{S}_{h}, \mathrm{~L}_{h}$, and $\mathrm{E}_{h}$. There are differences in the applicability of these operators concerning the types of elements and the ability to preserve Dirichlet boundary conditions. 
We will summarize this in Section 8. Before, in Section 7, we shall apply the operators $\mathrm{S}_{h}$ and $\mathrm{E}_{h}$ and derive finite element error estimates for the Poisson problem in certain domains with edges. The result can not be obtained using the nodal interpolation operator $\mathrm{I}_{h}$ or the original quasi-interpolation operators $\mathrm{C}_{h}, \mathrm{O}_{h}$, and $\mathrm{Z}_{h}$. This underlines the importance of this study.

Nevertheless, some questions need further research. First, the investigation in this paper is limited to domains of tensor product type. It is not straightforward to drop this assumption. Second, estimates of type $(1,1)$ are derived only for $\mathrm{L}_{h}$. This means, such an estimate is not available for three-dimensional "needle elements" $\left(h_{1} \sim h_{2} \ll h_{3}\right)$.

\section{Notation AND AUXiLiaRY RESUlts}

The notation $a \lesssim b$ and $a \sim b$ means the existence of positive constants $C_{1}$ and $C_{2}$ (which are independent of $\mathcal{T}_{h}$ and of the function under consideration) such that $a \leq C_{2} b$ and $C_{1} b \leq a \leq C_{2} b$, respectively.

Let $d$ be the space dimension and $x=\left(x_{1}, \ldots, x_{d}\right)$ the global Cartesian coordinate system. We use a multi-index notation with $\alpha:=\left(\alpha_{1}, \ldots, \alpha_{d}\right), \alpha_{i}$ non-negative integers,

$$
|\alpha|:=\sum_{i=1}^{d} \alpha_{i}, \quad x^{\alpha}:=x_{1}^{\alpha_{1}} \cdots x_{d}^{\alpha_{d}}, \text { and } D^{\alpha}:=\frac{\partial^{\alpha_{1}}}{\partial x_{1}^{\alpha_{1}}} \cdots \frac{\partial^{\alpha_{d}}}{\partial x_{d}^{\alpha_{d}}}
$$

$W^{\ell, p}(e)\left(\ell \in \mathbb{N}_{0}, p \in[1, \infty]\right)$ are the Sobolev spaces with

$$
\left\|v ; W^{\ell, p}(e)\right\|^{p}:=\sum_{|\alpha| \leq \ell} \int_{e}\left|D^{\alpha} v\right|^{p}, \quad\left|v ; W^{\ell, p}(e)\right|^{p}:=\sum_{|\alpha|=\ell} \int_{e}\left|D^{\alpha} v\right|^{p}
$$

for $p<\infty$ and the usual modification for $p=\infty$.

Finite elements $e \subset \mathbb{R}^{d}$ are defined via (a finite number of) reference element(s) $\hat{e} \subset \mathbb{R}^{d}$. In the cases of triangles $\left(\hat{e}:=\left\{\left(\hat{x}_{1}, \hat{x}_{2}\right) \in \mathbb{R}^{2}: 0<\hat{x}_{1}<1,0<\hat{x}_{2}<1-\hat{x}_{1}\right\}\right)$, rectangles $\left(\hat{e}:=\left\{\left(\hat{x}_{1}, \hat{x}_{2}\right) \in \mathbb{R}^{2}: 0<\hat{x}_{1}, \hat{x}_{2}<1\right\}\right)$, pentahedra $\left(\hat{e}:=\left\{\left(\hat{x}_{1}, \hat{x}_{2}, \hat{x}_{3}\right) \in \mathbb{R}^{3}: 0<\hat{x}_{1}, \hat{x}_{3}<1,0<\hat{x}_{2}<1-\hat{x}_{1}\right\}\right)$, and hexahedra $\left(\hat{e}:=\left\{\left(\hat{x}_{1}, \hat{x}_{2}, \hat{x}_{3}\right) \in\right.\right.$ $\left.\left.\mathbb{R}^{3}: 0<\hat{x}_{1}, \hat{x}_{2}, \hat{x}_{3}<1\right\}\right)$ it is sufficient to consider one unique $\hat{e}$. Only for tetrahedra we consider two reference elements: $\hat{e}:=\left\{\left(\hat{x}_{1}, \hat{x}_{2}, \hat{x}_{3}\right) \in \mathbb{R}^{3}: 0<\hat{x}_{1}<1,0<\hat{x}_{2}<1-\hat{x}_{1}, 0<\hat{x}_{3}<1-\hat{x}_{1}-\hat{x}_{2}\right\}$ for elements with a face parallel to the $x_{1}, x_{2}$-plane and $\hat{e}:=\left\{\left(\hat{x}_{1}, \hat{x}_{2}, \hat{x}_{3}\right) \in \mathbb{R}^{3}: 0<\hat{x}_{1}<1,0<\hat{x}_{2}<1-\hat{x}_{1}, \hat{x}_{1}<\hat{x}_{3}<1-\hat{x}_{2}\right\}$ for elements without such a face.

In this paper, we treat mainly meshes of tensor product type and tensor product meshes. The elements of these meshes are defined as follows.

Definition 1. An affine finite element is called element of tensor product type, when the transformation of a reference element $\hat{e}$ to the element $e$ has (block) diagonal form,

$$
\begin{aligned}
& \left(\begin{array}{l}
x_{1} \\
x_{2}
\end{array}\right)=\left(\begin{array}{cc} 
\pm h_{1, e} & 0 \\
0 & \pm h_{2, e}
\end{array}\right)\left(\begin{array}{l}
\hat{x}_{1} \\
\hat{x}_{2}
\end{array}\right)+b_{e} \quad \text { for } d=2,
\end{aligned}
$$

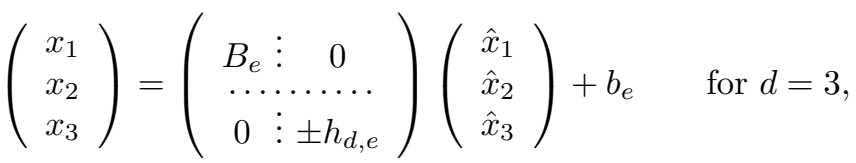

where $b_{e} \in \mathbb{R}^{d}$ and $B_{e} \in \mathbb{R}^{2 \times 2}$ with

$$
\left|\operatorname{det} B_{e}\right| \sim h_{1, e}^{2}, \quad\left\|B_{e}\right\| \sim h_{1, e}, \quad\left\|B_{e}^{-1}\right\| \sim h_{1, e}^{-1}
$$

In this way the element sizes $h_{1, e}, \ldots, h_{d, e}$ are implicitly defined. Note that (2.3) yields $h_{1, e} \sim h_{2, e}$ for threedimensional elements. Up to now we did not assume a relation between $h_{1, e}$ and $h_{d, e}$. But in Sections 4 and 6 


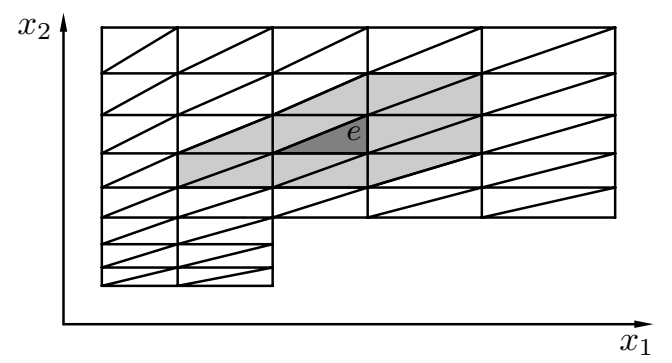

Figure 2. Illustration of a mesh of tensor product type in two dimensions and of the patch $S_{e}$.

we will consider the case $h_{1, e} \lesssim h_{d, e}$ (interesting is $\left.h_{1, e}=o\left(h_{d, e}\right)\right)$ and in Section 5 we will examine $h_{d, e} \lesssim h_{1, e}$. Note further that under these assumptions the triangles/tetrahedra can be grouped into pairs/triples which form a rectangle/pentahedron of tensor product type. We will use this property in Section 4.

Definition 2. An affine finite element $e \subset \mathbb{R}^{d}$ is called tensor product element, when transformation (2.2) is reduced to

$$
x_{i}=h_{i, e} \hat{x}_{i}+b_{i, e}, \quad i=1, \ldots, d .
$$

In two dimensions there is no difference between tensor product elements and elements of tensor product type. But in three dimensions we admit independent mesh sizes $h_{1, e}, h_{2, e}$, and $h_{3, e}$, so that a tensor product element is not necessarily a special case of an element of tensor product type.

We demand that there is no abrupt change in the element sizes, that means, the relation

$$
h_{i, e} \sim h_{i, e^{\prime}} \quad \text { for all } e^{\prime} \text { with } \quad \bar{e} \cap \overline{e^{\prime}} \neq \emptyset
$$

holds for $i=1, \ldots, d$. In view of $(2.5)$ and because most considerations in this paper are local, we will often omit the second subscript.

The set of shape functions $\mathcal{P}_{k, e}$,

$$
\mathcal{P}_{k, e} \supset \mathcal{P}_{k}^{d}:=\left\{\sum_{|\alpha| \leq k} a_{\alpha} x^{\alpha} ; \quad x=\left(x_{1}, \ldots, x_{d}\right), a_{\alpha} \in \mathbb{R}\right\}
$$

is defined as usual, that means, $\mathcal{P}_{k, e}=\mathcal{P}_{k}^{d}$ for the simplicial elements, and

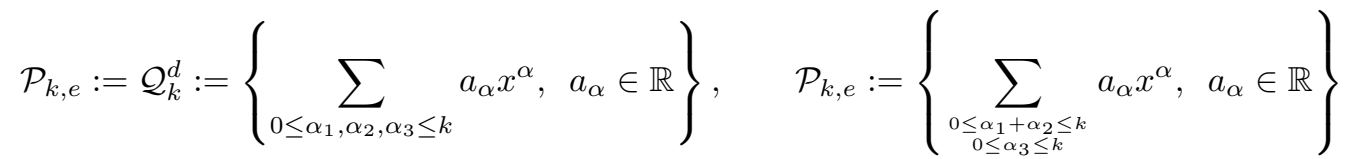

for quadrilateral/hexahedral elements and for pentahedral elements, respectively. Moreover, for a simple notation later on we define $\mathcal{P}_{-1}^{d}:=\{0\}$.

Let $V_{h}:=\left\{v_{h} \in W^{1,2}(\Omega):\left.v_{h}\right|_{e} \in \mathcal{P}_{k, e}\right.$ for all $\left.e \in \mathcal{T}_{h}\right\}$ be the finite element space, a space of piecewise polynomial functions on the family of meshes under consideration. 
Finally, denote by

$$
S_{e}:=\operatorname{int} \bigcup\left\{\overline{e^{\prime}}: e^{\prime} \in \mathcal{T}_{h}, \overline{e^{\prime}} \cap \bar{e} \neq \emptyset\right\}
$$

the patch of elements around $e$, see also the illustration for a general mesh in Figure 2. Moreover, we denote uniformly in the whole paper by

$$
\begin{aligned}
& X_{i} \quad \text { the nodes of the mesh, } i \in I \text {, } \\
& \varphi_{i} \text { the nodal shape functions, } \varphi_{i}\left(X_{j}\right)=\delta_{i j} \text {, } \\
& \sigma_{i} \text { a subdomain related to } X_{i} \text { (different for } \mathrm{C}_{h}, \mathrm{O}_{h}, \mathrm{Z}_{h}, \mathrm{~S}_{h}, \mathrm{~L}_{h} \text {, and } \mathrm{E}_{h} \text { ), } \\
& k \text { the degree of the shape functions in the sense of (2.6), } \\
& \Pi_{\sigma_{i}} \text { the projection operator } L^{2}\left(\sigma_{i}\right) \rightarrow \mathcal{P}_{k, \sigma_{i}} \text {, } \\
& \mathrm{I}_{h} \quad \text { the nodal interpolation operator, } \\
& \mathrm{Q}_{h} \text { a general quasi-interpolation operator, } \\
& \mathrm{C}_{h} \text { the } \text { Clément operator, } \\
& \mathrm{O}_{h} \text { the quasi-interpolation operator introduced by } \underline{\text { Oswald, }} \\
& \mathrm{Z}_{h} \text { the original Scott- } \underline{Z} \text { hang operator, } \\
& \mathrm{S}_{h} \text { the modified Scott-Zhang operator using small edges(2D)/faces(3D), } \\
& \mathrm{L}_{h} \text { the modified Scott-Zhang operator using large edges(2D)/faces(3D), } \\
& \mathrm{E}_{h} \text { the modified Scott-Zhang operator using long edges (3D). }
\end{aligned}
$$

We will prove now a lemma which is useful in several proofs of this paper. The lemma has similarities to the Bramble-Hilbert theory which was developed in $[16,17]$ for isotropic elements and extended in [4] to anisotropic elements. Here, the difference is that (in general) $S_{e}$ can not be transformed by an affine mapping to a reference configuration $\hat{S}$. The isotropic version of Lemma 1 is proved in [30] using results from [21] and can easily be generalized to our case.

Lemma 1. For any $u \in W^{\ell, p}\left(S_{e}\right)$ there exists a polynomial $w \in \mathcal{P}_{\ell-1}^{d}$ such that

$$
\sum_{|\alpha| \leq \ell-m} h^{\alpha}\left|D^{\alpha}(u-w) ; W^{m, p}\left(S_{e}\right)\right| \lesssim \sum_{|\alpha|=\ell-m} h^{\alpha}\left|D^{\alpha} u ; W^{m, p}\left(S_{e}\right)\right|
$$

for all $m=0, \ldots, \ell$.

Proof. By the change of variables $x_{i}=\tilde{x}_{i} h_{i}$ we transform $S_{e}$ to $\tilde{S}_{e}$. According to $(2.5)$ and the tensor product character of our mesh we realize that $\tilde{S}_{e}$ has a diameter of order one. Moreover, $\tilde{S}_{e}$ is star-shaped with respect to a ball $B_{1}$ with diam $B_{1} \sim 1$, or $\tilde{S}_{e}$ is at least the union of a finite collection of (overlapping) domains $\tilde{S}_{e, j}$ that are star-shaped with respect to a balls $B_{j}$ with $\operatorname{diam} B_{j} \sim 1$. Let $B \subset \tilde{S}_{e}$ be any ball with $\operatorname{diam} B \sim 1$, choose a function $\phi \in C_{0}^{\infty}(B)$ with integral one, and define

$$
\tilde{w}(\tilde{x}):=\sum_{|\alpha| \leq \ell-1} \int_{B} \phi(\tilde{y}) \cdot\left(\tilde{D}^{\alpha} \tilde{u}\right)(\tilde{y}) \cdot \frac{(\tilde{x}-\tilde{y})^{\alpha}}{\alpha !} \mathrm{d} \tilde{y} \in \mathcal{P}_{\ell-1}^{d}
$$

$\tilde{x}=\left(\tilde{x}_{1}, \ldots, \tilde{x}_{d}\right), \tilde{y}=\left(\tilde{y}_{1}, \ldots, \tilde{y}_{d}\right), \alpha !=\alpha_{1} ! \cdots \alpha_{d} !$. We can now apply Theorem 4.2 of $[21]$ with $\mathcal{A}=\left\{\alpha \in \mathbb{N}_{0}^{d}:\right.$ $|\alpha|=\ell\}$, and obtain for all $\beta$ with $|\beta|=m, 0 \leq m \leq \ell-1$,

$$
\left\|\tilde{D}^{\beta}(\tilde{u}-\tilde{w}) ; W^{\ell-m-1, p}\left(\tilde{S}_{e}\right)\right\| \lesssim\left|\tilde{D}^{\beta} \tilde{u} ; W^{\ell-m, p}\left(\tilde{S}_{e}\right)\right|
$$


By transforming this estimate to $S_{e}$ and summing up over all $\beta$ we conclude

$$
\begin{aligned}
\sum_{|\alpha| \leq \ell-m-1} h^{\alpha}\left\|D^{\alpha+\beta}(u-w) ; L^{p}\left(S_{e}\right)\right\| & \lesssim \sum_{|\alpha|=\ell-m} h^{\alpha}\left\|D^{\alpha+\beta} u ; L^{p}\left(S_{e}\right)\right\|, \\
\sum_{|\alpha| \leq \ell-m-1} h^{\alpha}\left|D^{\alpha}(u-w) ; W^{m, p}\left(S_{e}\right)\right| & \lesssim \sum_{|\alpha|=\ell-m} h^{\alpha}\left|D^{\alpha} u ; W^{m, p}\left(S_{e}\right)\right| .
\end{aligned}
$$

Because $D^{\gamma} w=0$ for $|\gamma|=\ell$ the sum on the left-hand side can be extended to $|\alpha| \leq \ell-m$.

Corollary 2. Let $m_{1}+m_{2}=m \leq \ell$. For any $u \in W^{\ell, p}\left(S_{e}\right)$ there exists a polynomial $w \in \mathcal{P}_{m-1}^{d}$ such that

$$
\sum_{|\alpha| \leq m_{2}} \sum_{|\beta| \leq \ell-m} h^{\alpha+\beta}\left|D^{\alpha+\beta}(u-w) ; W^{m_{1}, p}\left(S_{e}\right)\right| \lesssim \sum_{|\alpha|=m_{2}} \sum_{|\beta| \leq \ell-m} h^{\alpha+\beta}\left|D^{\alpha+\beta} u ; W^{m_{1}, p}\left(S_{e}\right)\right| .
$$

Proof. We reformulate the left-hand side and split it in two terms.

$$
\begin{aligned}
\sum_{|\alpha| \leq m_{2}} \sum_{|\beta| \leq \ell-m} h^{\alpha+\beta}\left|D^{\alpha+\beta}(u-w) ; W^{m_{1}, p}\left(S_{e}\right)\right| \sim \sum_{|\delta| \leq \ell-m_{1}} h^{\delta}\left|D^{\delta}(u-w) ; W^{m_{1}, p}\left(S_{e}\right)\right| \\
=\sum_{|\delta| \leq m_{2}} h^{\delta}\left|D^{\delta}(u-w) ; W^{m_{1}, p}\left(S_{e}\right)\right|+\sum_{m_{2}<|\delta| \leq \ell-m_{1}} h^{\delta}\left|D^{\delta}(u-w) ; W^{m_{1}, p}\left(S_{e}\right)\right| .
\end{aligned}
$$

In view of $m_{2}=m-m_{1}$, the first term can be estimated via Lemma 1 . The second term contains only derivatives of order higher than $m$, that means that $w$ plays no role. Consequently, $w$ can be chosen such that

$$
\begin{aligned}
& \sum_{|\alpha| \leq m_{2}} \sum_{|\beta| \leq \ell-m} h^{\alpha+\beta}\left|D^{\alpha+\beta}(u-w) ; W^{m_{1}, p}\left(S_{e}\right)\right| \\
& \lesssim \sum_{|\delta|=m_{2}} h^{\delta}\left|D^{\delta} u ; W^{m_{1}, p}\left(S_{e}\right)\right|+\sum_{m_{2}<|\delta| \leq \ell-m_{1}} h^{\delta}\left|D^{\delta} u ; W^{m_{1}, p}\left(S_{e}\right)\right| \\
& \lesssim \sum_{|\alpha|=m_{2}} h^{\alpha}\left|D^{\alpha} u ; W^{m_{1}, p}\left(S_{e}\right)\right|+\sum_{|\alpha|=m_{2}} \sum_{1 \leq|\beta| \leq \ell-m} h^{\alpha+\beta}\left|D^{\alpha+\beta} u ; W^{m_{1}, p}\left(S_{e}\right)\right|
\end{aligned}
$$

and the corollary is proved.

\section{The original Scott-Zhang operator $Z_{h}$}

In this section we will recall the operator $\mathrm{Z}_{h}$ defined by Scott and Zhang [30] and examine to what extent anisotropic error estimates can be derived by simply carrying out the transformations more carefully. We will see that estimates of type $(0, \ell)$ are valid, but modifications of the operator are necessary for estimates of derivatives of the approximation error.

As introduced in Section 1 we define $\mathrm{Z}_{h} u$ via numbers $a_{i}=\left(\Pi_{\sigma_{i}} u\right)\left(X_{i}\right)$, where $\Pi_{\sigma_{i}}$ is a projection operator with respect to a certain subdomain $\sigma_{i}, i \in I$. The subdomains $\sigma_{i}$ are chosen by the following rules (see also Fig. 3 for the case of triangles).

- If the node $X_{i}$ is an interior point of an element $e \subset \mathcal{T}_{h}$ then $\sigma_{i}:=e$.

- Otherwise $X_{i}$ is a boundary point of one or more elements $e \subset \mathcal{T}_{h}$, and $\sigma_{i}$ is chosen as some $(d-1)$ dimensional edge/face $\varsigma$ of one of these elements:

- If there is an edge/face $\varsigma$ so that $X_{i}$ is an interior point of $\varsigma$, then $\sigma_{i}$ is uniquely determined by $\sigma_{i}:=\varsigma$.

- If not, then $\sigma_{i}$ is taken as one of the edges/faces with $X_{i} \in \bar{\varsigma}$. However, we restrict this choice in the case $X_{i} \in \partial \Omega$ by demanding $\sigma_{i} \subset \partial \Omega$ then. 


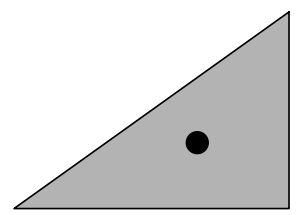

(a) $X_{i}$ is an interior point of an element.

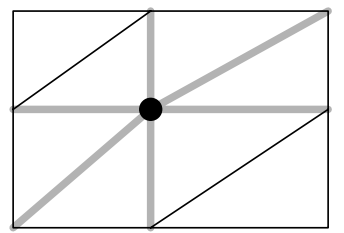

(c) $X_{i}$ is a vertex within the domain (here: 6 possibilities for $\sigma_{i}$ ).

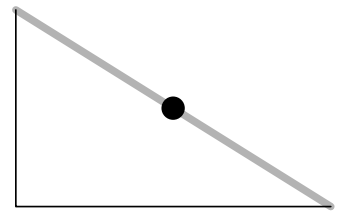

(b) $X_{i}$ is an interior point of an edge.

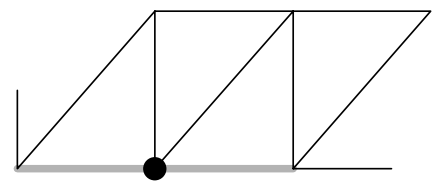

(d) $X_{i}$ is a vertex at the boundary (2 possibilities for $\sigma_{i}$ ).

Figure 3. Choice of $\sigma_{i}$ in dependence on $X_{i}$ for the definition of $\mathrm{Z}_{h}$.

The $L^{2}\left(\sigma_{i}\right)$-projection $\left.\Pi_{\sigma_{i}} u \in V_{h}\right|_{\sigma_{i}}$ is defined by

$$
\left\|u-\Pi_{\sigma_{i}} u ; L^{2}\left(\sigma_{i}\right)\right\|=\min _{\left.v \in V_{h}\right|_{\sigma_{i}}}\left\|u-v ; L^{2}\left(\sigma_{i}\right)\right\| .
$$

An explicit representation of $\left(\Pi_{\sigma_{i}} u\right)\left(X_{i}\right)$ can be given by introducing the (unique) function $\left.\psi_{i} \in V_{h}\right|_{\sigma_{i}}$ with

$$
\int_{\sigma_{i}} \psi_{i} \varphi_{j}=\delta_{i j} \quad \text { for all } j \in I .
$$

Then one finds easily that

$$
\left(\Pi_{\sigma_{i}} u\right)\left(X_{i}\right)=\int_{\sigma_{i}} u \psi_{i} .
$$

To see this recall that a projection operator $P: X \rightarrow Y \subset X$ can be defined via $P u=\sum_{j}\left(u, \psi_{j}\right)_{X} \varphi_{j}$ where $\left\{\varphi_{j}\right\}$ is a basis in $Y$ and $\left\{\psi_{j}\right\}$ is the corresponding biorthogonal basis with respect to the scalar product $(., .)_{X}$ in $X$. As already mentioned in Section 1 , see (1.4) and (1.6), the Scott-Zhang operator $Z_{h}$ is now defined as

$$
\mathrm{Z}_{h} u:=\sum_{i}\left(\Pi_{\sigma_{i}} u\right)\left(X_{i}\right) \cdot \varphi_{i}=\sum_{i}\left(\int_{\sigma_{i}} u \psi_{i}\right) \cdot \varphi_{i} .
$$

Though $\Pi_{\sigma_{i}}$ is defined by (3.1) for $u \in L^{2}\left(\sigma_{i}\right)$, this approach can be extended to functions $u \in L^{1}\left(\sigma_{i}\right)$ because the polynomial function $\psi_{i}$ is from $L^{\infty}\left(\sigma_{i}\right)$ so the integral in (3.3) is finite. That means that the approximation operator $\mathrm{Z}_{h}: W^{\ell, p}(\Omega) \rightarrow V_{h}$ can be defined for

$$
\ell \geq 1 \quad \text { for } p=1, \quad \ell>\frac{1}{p} \quad \text { otherwise. }
$$

The restrictions to $\ell$ and $p$ in (3.5) follow from a trace theorem and guarantee that $\left.u\right|_{\sigma_{i}} \in L^{1}\left(\sigma_{i}\right)$ also for $(d-1)$-dimensional $\sigma_{i}$. In this paper, we consider only integer $\ell$, therefore (3.5) is equivalent to

$$
\ell \geq 1, \quad p \in[1, \infty]
$$


Note further that the approximation operator $\mathrm{Z}_{h}$ does not only preserve homogeneous Dirichlet boundary conditions but also inhomogeneous conditions $u=g$ on $\partial \Omega$ (at least in the sense of $L^{1}(\partial \Omega)$ ) if $\left.g \in V_{h}\right|_{\partial \Omega}$.

For isotropic simplicial elements $e\left(h_{1} \sim \ldots \sim h_{d}\right)$ Scott and Zhang proved the following stability and approximation result [30]: If $1 \leq \ell \leq k+1$ and $p \in[1, \infty]$ then the estimates

$$
\begin{aligned}
\left|\mathrm{Z}_{h} u ; W^{m, q}(e)\right| & \lesssim(\text { meas } e)^{1 / q-1 / p} \sum_{j=0}^{\ell} h_{1}^{j-m}\left|u ; W^{j, p}\left(S_{e}\right)\right| \\
\left|u-\mathrm{Z}_{h} u ; W^{m, p}(e)\right| & \lesssim h_{1}^{\ell-m}\left|u ; W^{\ell, p}\left(S_{e}\right)\right|
\end{aligned}
$$

hold for $0 \leq m \leq \ell$. Recall that $k$ corresponds to the degree of the polynomials, see (2.6). Recall also the definition of $S_{e}$ from (2.7) and note that $\sigma_{i} \subset S_{e}$ for all $i$ with $X_{i} \in \bar{e}$.

The anisotropic estimate corresponding to (3.7) would be

$$
\left|u-\mathrm{Z}_{h} u ; W^{m, p}(e)\right| \lesssim \sum_{|\alpha|=\ell-m} h^{\alpha}\left|D^{\alpha} u ; W^{m, p}\left(S_{e}\right)\right|
$$

We prove now that this estimate is valid for $m=0$. This result is restricted here to meshes of tensor product type but it is not restricted to simplicial elements.

Theorem 3. On anisotropic meshes of tensor product type the Scott-Zhang approximation operator $\mathrm{Z}_{h}$ satisfies the following stability and approximation error estimates of type $(0, \ell)$ :

$$
\begin{aligned}
\left\|\mathrm{Z}_{h} u ; L^{q}(e)\right\| & \lesssim(\text { meas } e)^{1 / q-1 / p} \sum_{|\alpha| \leq \ell} h^{\alpha}\left\|D^{\alpha} u ; L^{p}\left(S_{e}\right)\right\|, \\
\left\|u-\mathrm{Z}_{h} u ; L^{q}(e)\right\| & \lesssim(\text { meas } e)^{1 / q-1 / p} \sum_{|\alpha|=\ell} h^{\alpha}\left\|D^{\alpha} u ; L^{p}\left(S_{e}\right)\right\|,
\end{aligned}
$$

$\ell=1, \ldots, k+1$, provided that $u \in W^{\ell, p}\left(S_{e}\right)$. For $(3.10)$ the numbers $p, q \in[1, \infty]$ and $\ell \in \mathbb{N}$ must be such that $W^{\ell, p}(e) \hookrightarrow L^{q}(e)$.

Proof. We start by concluding from $\int_{\sigma_{i}} \varphi_{i} \psi_{i}=1$ and $\left\|\varphi_{i} ; L^{\infty}\left(\sigma_{i}\right)\right\|=1$ that

$$
\left\|\psi_{i} ; L^{\infty}\left(\sigma_{i}\right)\right\| \sim\left(\operatorname{meas} \sigma_{i}\right)^{-1}
$$

Using the definition of $\mathrm{Z}_{h} u$ we find with (3.11) that

$$
\begin{aligned}
\left\|\mathrm{Z}_{h} u ; L^{q}(e)\right\| & \leq \sum_{i \in I_{e}}\left\|\varphi_{i} \int_{\sigma_{i}} u \psi_{i} ; L^{q}(e)\right\| \\
& \leq(\operatorname{meas} e)^{1 / q} \sum_{i \in I_{e}}\left|\int_{\sigma_{i}} u \psi_{i}\right| \\
& \lesssim(\operatorname{meas} e)^{1 / q} \sum_{i \in I_{e}}\left(\operatorname{meas} \sigma_{i}\right)^{-1}\left\|u ; L^{1}\left(\sigma_{i}\right)\right\|,
\end{aligned}
$$

where $I_{e}$ is the set of nodes contained in $\bar{e}$. If $\sigma_{i}$ has the same dimension as $e$ (that means $X_{i}$ is an inner node of $e$ and $\sigma_{i}=e$ ) then we use the Hölder inequality and find

$$
\begin{aligned}
\left\|u ; L^{1}\left(\sigma_{i}\right)\right\| & \leq(\text { meas } e)^{1-1 / p}\left\|u ; L^{p}\left(\sigma_{i}\right)\right\| \\
& \lesssim \operatorname{meas} \sigma_{i}(\operatorname{meas} e)^{-1 / p}\left\|u ; L^{p}\left(S_{e}\right)\right\| .
\end{aligned}
$$




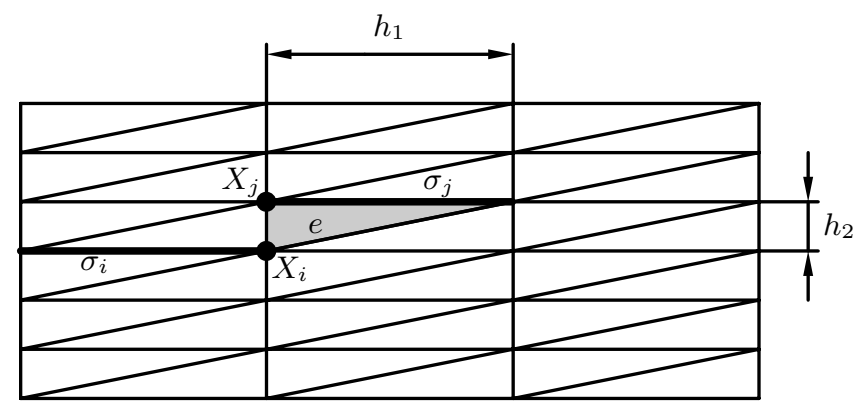

FIGURE 4. Illustration of the counterexample.

If $\sigma_{i}$ has lower dimension we use the trace theorem $W^{\ell, p}\left(S_{e}\right) \hookrightarrow W^{\ell, p}\left(e^{\prime}\right) \hookrightarrow L^{1}\left(\sigma_{i}\right)\left(e^{\prime} \subset S_{e}\right.$ is an element with $\left.\sigma_{i} \subset \overline{e^{\prime}}\right)$ in the form

$$
\left\|u ; L^{1}\left(\sigma_{i}\right)\right\| \lesssim \text { meas } \sigma_{i}(\text { meas } e)^{-1 / p} \sum_{|\alpha| \leq \ell} h^{\alpha}\left\|D^{\alpha} u ; L^{p}\left(S_{e}\right)\right\|
$$

which holds for $\ell \geq 1$. Combining the last three estimates we obtain the stability estimate (3.9). From this we derive for any $w \in \mathcal{P}_{\ell-1}^{d} \subset \mathcal{P}_{k}^{d}$

$$
\begin{aligned}
\left\|u-\mathrm{Z}_{h} u ; L^{q}(e)\right\| & \leq\left\|u-w ; L^{q}(e)\right\|+\left\|\mathrm{Z}_{h}(u-w) ; L^{q}(e)\right\| \\
& \lesssim(\operatorname{meas} e)^{1 / q-1 / p} \sum_{|\alpha| \leq \ell} h^{\alpha}\left\|D^{\alpha}(u-w) ; L^{p}\left(S_{e}\right)\right\|
\end{aligned}
$$

where we used the embedding $W^{\ell, p}(e) \hookrightarrow L^{q}(e)$. With Lemma 1 we conclude (3.10).

By the following example we show that estimate (3.8) does not hold for $m \geq 1$ in the general setting of $\sigma_{i}$ as introduced above.

Example 1. In this example we will show that (3.8) does in general not hold in the case $m=k=1$ and the whole range of $\ell$, namely $\ell=1,2$. Consider the situation as illustrated in Figure 4 , and let $u=u\left(x_{1}\right)$ be any function which is independent of the variable $x_{2}$. This leads to $a_{i} \neq a_{j}$, where $a_{i}$ and $a_{j}$ are independent of $h_{2}$, that means

$$
\left.\frac{\partial \mathrm{Z}_{h} u}{\partial x_{2}}\right|_{e}=h_{2}^{-1} f\left(u, x_{1}, h_{1}\right)
$$

with a certain function $f$. In view of $\partial u / \partial x_{2}=0$ we obtain

$$
\begin{aligned}
\left|u-\mathrm{Z}_{h} u ; W^{1, p}(e)\right| & \geq\left\|\frac{\partial \mathrm{Z}_{h} u}{\partial x_{2}}, L^{p}(e)\right\|=h_{2}^{-1+1 / p} F\left(u, h_{1}\right), \\
\sum_{|\alpha|=\ell-1} h^{\alpha}\left|D^{\alpha} u ; W^{1, p}\left(S_{e}\right)\right| & =h_{1}^{\ell-1}\left\|\frac{\partial^{\ell} u}{\partial x_{1}^{\ell}}, L^{p}\left(S_{e}\right)\right\|=h_{2}^{1 / p} G\left(u, h_{1}\right) .
\end{aligned}
$$

Consequently, for $f\left(u, x_{1}, h_{1}\right) \neq 0$ (which is the case in general) and $h_{2}=h_{1}^{s}$ with sufficiently large $s$ (depending on $u$ ) estimate (3.8) can not be satisfied.

For this example the following points were essential:

1. Long edges are chosen for $\sigma_{i}$. 
2. $X_{i}$ and $X_{j}$ have the same $x_{1}$-coordinate but the projections of $\sigma_{i}$ and $\sigma_{j}$ on the $x_{1}$-axis are different.

Since we have some freedom in the choice of $\sigma_{i}$ we will investigate in the next two sections the operator in the cases where one of these points is avoided. In Section 4 we will use short edges (2D) or small faces (3D) as $\sigma_{i}$. Large sides with identical projection are chosen in Section 5. The resulting operators will be denoted by $\mathrm{S}_{h}$ (small sides) and $\mathrm{L}_{h}$ (large sides).

Having now an idea which choice of $\sigma_{i}$ could work, we want to point out that the desired error estimate cannot be obtained with the original proof of [30]. We can see this from the following two examples.

Example 2. The proof of Theorem 3 followed essentially the steps of the proof in [30]. Let us see which result we obtain for a derivative. Consider an element $e \subset \mathbb{R}^{2}$ of a mesh of tensor product type, a function $u \in W^{\ell, p}\left(S_{e}\right), \ell \in\{1,2\}$, the polynomial degree $k=1$ and a multi-index $\gamma$ with $|\gamma|=1$. Let all $\sigma_{i}$ be defined as short edges. Then we get by following the proof of Theorem 3

$$
\begin{aligned}
\left\|D^{\gamma} \mathrm{S}_{h} u ; L^{q}(e)\right\| & \leq \sum_{i \in I_{e}}\left\|D^{\gamma} \varphi_{i} ; L^{q}(e)\right\|\left|\int_{\sigma_{i}} u \psi_{i}\right| \\
& \lesssim h^{-\gamma}(\operatorname{meas} e)^{1 / q} \sum_{i \in I_{e}}\left(\operatorname{meas} \sigma_{i}\right)^{-1}\left\|u ; L^{1}\left(\sigma_{i}\right)\right\| \\
& \lesssim h^{-\gamma}(\operatorname{meas} e)^{1 / q} \sum_{i \in I_{e}}(\operatorname{meas} e)^{-1 / p} \sum_{|\alpha| \leq \ell} h^{\alpha}\left\|D^{\alpha} u ; L^{p}\left(S_{e}\right)\right\| \\
& \sim h^{-\gamma}(\operatorname{meas} e)^{1 / q-1 / p} \sum_{|\alpha| \leq \ell} h^{\alpha}\left\|D^{\alpha} u ; L^{p}\left(S_{e}\right)\right\| .
\end{aligned}
$$

For estimating the error $D^{\gamma}\left(u-\mathrm{S}_{h} u\right)$ we apply this estimate to $u-w$ instead of $u$, with $w \in \mathcal{P}_{\ell-1}^{d}$. By applying Lemma 1 we get

$$
\left\|D^{\gamma}\left(u-\mathrm{S}_{h} u\right) ; L^{q}(e)\right\| \lesssim h^{-\gamma}(\text { meas } e)^{1 / q-1 / p} \sum_{|\alpha|=\ell} h^{\alpha}\left\|D^{\alpha} u ; L^{p}\left(S_{e}\right)\right\|
$$

Let $h_{1} \ll h_{2}, \gamma=(1,0)$, then one term at the right-hand side is $h_{1}^{-1} h_{2}^{\ell}\left\|D^{(0,2)} u ; L^{p}\left(S_{e}\right)\right\|$ which may become arbitrary large. Therefore we do not obtain estimate (3.8) with the original proof, but only a sub-optimal right-hand side as in (3.14).

Example 3. Let us perform a backward analysis. Assume that (3.8) is the appropriate estimate for an element $e$ of a mesh of tensor product type with an arbitrary $h_{1} \ll h_{2}$. For $m=1, \ell=2$, we have in particular

$$
\begin{aligned}
\left\|D^{(1,0)}\left(u-\mathrm{S}_{h} u\right) ; L^{p}(e)\right\| & \lesssim \sum_{|\alpha|=1} h^{\alpha}\left|D^{\alpha} u ; W^{1, p}\left(S_{e}\right)\right| \\
& \sim \sum_{|\alpha|=1} h^{\alpha}\left\|D^{\alpha+(1,0)} u ; L^{p}\left(S_{e}\right)\right\|+h_{2}\left\|D^{(0,2)} u ; L^{p}\left(S_{e}\right)\right\| .
\end{aligned}
$$

Change the variables via $x_{i}=\tilde{x}_{i} h_{i}, i=1,2$, to obtain an estimate for an element $\tilde{e}$ with diam $\tilde{e} \sim \varrho_{\tilde{e}} \sim 1$. The estimate (3.15) transforms to

$$
\begin{aligned}
h_{1}^{-1}\left\|\tilde{D}^{(1,0)}\left(\tilde{u}-\mathrm{S}_{h} \tilde{u}\right) ; L^{p}(\tilde{e})\right\| & \lesssim \sum_{|\alpha|=1} h_{1}^{-1}\left\|\tilde{D}^{\alpha+(1,0)} \tilde{u} ; L^{p}\left(\tilde{S}_{e}\right)\right\|+h_{2}^{-1}\left\|\tilde{D}^{(0,2)} \tilde{u} ; L^{p}\left(\tilde{S}_{e}\right)\right\|, \\
\left\|\tilde{D}^{(1,0)}\left(\tilde{u}-\mathrm{S}_{h} \tilde{u}\right) ; L^{p}(\tilde{e})\right\| & \lesssim \sum_{|\alpha|=1}\left\|\tilde{D}^{\alpha+(1,0)} \tilde{u} ; L^{p}\left(\tilde{S}_{e}\right)\right\|+h_{2}^{-1} h_{1}\left\|\tilde{D}^{(0,2)} \tilde{u} ; L^{p}\left(\tilde{S}_{e}\right)\right\| .
\end{aligned}
$$


For this estimate to be satisfied for arbitrary $h_{1}=o\left(h_{2}\right)$ we have to show

$$
\begin{aligned}
\left\|\tilde{D}^{\gamma}\left(\tilde{u}-\mathrm{S}_{h} \tilde{u}\right) ; L^{p}(\tilde{e})\right\| & \lesssim\left|\tilde{D}^{\gamma} \tilde{u} ; W^{1, p}\left(\tilde{S}_{e}\right)\right|, \\
\left\|D^{\gamma}\left(u-\mathrm{S}_{h} u\right) ; L^{p}(e)\right\| & \lesssim \sum_{|\alpha|=1} h^{\alpha}\left\|D^{\alpha+\gamma} u ; L^{p}\left(S_{e}\right)\right\|,
\end{aligned}
$$

at least for $\gamma=(1,0)$. Otherwise the estimate is not invariant with respect to scaling. If we want to derive the error estimate by using the stability estimate as in the proof of Theorem 3 , we must prove

$$
\left\|D^{\gamma} \mathrm{S}_{h} u ; L^{q}(e)\right\| \lesssim(\text { meas } e)^{1 / q-1 / p} \sum_{|\alpha| \leq \ell-|\gamma|} h^{\alpha}\left|D^{\alpha} u ; W^{|\gamma|, p}\left(S_{e}\right)\right| .
$$

We have seen in the examples that choosing appropriate $\sigma_{i}$ is not enough. We need also a refined proof for obtaining anisotropic estimates for derivatives of the interpolation error. We will develop such refined proofs for general $k, \ell, m$, in the next sections. However, we need in all cases that all $\sigma_{i}, i \in I$, are parallel. Therefore we are restricted to meshes of tensor product type (introduced in Def. 1 and investigated in Sects. 4 and 5) or to tensor product meshes (introduced in Def. 2 and investigated in Sect. 6). The proof for more general meshes is still open.

In the remaining part of this section we will discuss to what extent the previous results carry over to the operators $\mathrm{C}_{h}$ and $\mathrm{O}_{h}$ which were considered by Clément [19] and Oswald [28] for isotropic meshes. Recall from the Introduction that the difference between $\mathrm{Z}_{h}, \mathrm{C}_{h}$, and $\mathrm{O}_{h}$ is only in the definition of the subdomains $\sigma_{i}$. In particular, $\sigma_{i}$ is $d$-dimensional for $\mathrm{C}_{h}$ and $\mathrm{O}_{h}$ and for all $i \in I$.

For $\mathrm{O}_{h}$ one can verify easily that all results in this section remain true, except that Dirichlet boundary conditions are not satisfied. Moreover, Condition (3.5) can even be omitted; the operator is defined for all $u \in L^{1}(\Omega)$. Therefore estimates $(3.6,3.7,3.9,3.10)$ hold for $\ell=0$ as well. Example 1 can be modified in the obvious way. ( $\mathrm{Z}_{h}$ has to be substituted by $\mathrm{O}_{h}$ in all relations.)

For the Clément operator $\mathrm{C}_{h}$, one has to decide whether $\Pi_{\sigma_{i}}$ should be defined as in (1.5) or (1.7). In both cases the same estimates as for $\mathrm{O}_{h}$ can be proved. Note that we used in the proof only $\mathrm{C}_{h} w=w$ for $w \in \mathcal{P}_{k}^{d}$ which is satisfied. As discussed already in the Introduction, $\mathrm{C}_{h} v_{h}=v_{h}$ is in general not satisfied for $v_{h} \in V_{h}$.

Siebert [32] and Kunert [24] derived also some results for the operator $\mathrm{C}_{h}$ for anisotropic meshes. However, they considered only the case $k=1, p=2$, and only subsets $H_{\mathcal{T}}^{1}(\Omega) \subset W^{1,2}(\Omega)$ of so-called mesh adapted functions. This allows them to prove global results of the form

$$
\begin{aligned}
\sum_{e} \varrho_{e}^{-1}\left\|v-\mathrm{C}_{h} v, L^{2}(e)\right\| & \lesssim\left|v ; W^{1,2}(\Omega)\right|, \\
\left.\sum_{e} h_{i, e} \varrho_{e}^{-1} \| \frac{\partial}{\partial x_{i}}\left(v-\mathrm{C}_{h} v\right)\right), L^{2}(e) \| & \lesssim\left|v ; W^{1,2}(\Omega)\right|, \quad i=1, \ldots, d,
\end{aligned}
$$

where $\varrho_{e} \sim \min _{j=1, \ldots, d} h_{j, e}$. Using these estimates they prove asymptotic properties of a posteriori error estimators. For $v$ they insert the (exact) finite element error $u-u_{h}$. Unfortunately, the condition $u-u_{h} \in H_{\mathcal{T}}^{1}(\Omega)$ can not be proved/tested in general.

To satisfy Dirichlet boundary conditions all the authors $[19,24,32]$ considered a modification of $\mathrm{C}_{h}$ near the boundary which is small enough to keep the approximation order.

\section{The operator $\mathrm{S}_{h}$ : A modification of $\mathrm{Z}_{h}$ BY CHOOSING SMALL Sides}

\subsection{Stability and approximation in classical Sobolev spaces}

In this section we will investigate the operator $S_{h}$ which was first introduced in Section 3, after Example 1. Throughout the section we assume that $e$ is an element of tensor product type, see Definition 1 in Section 2. 


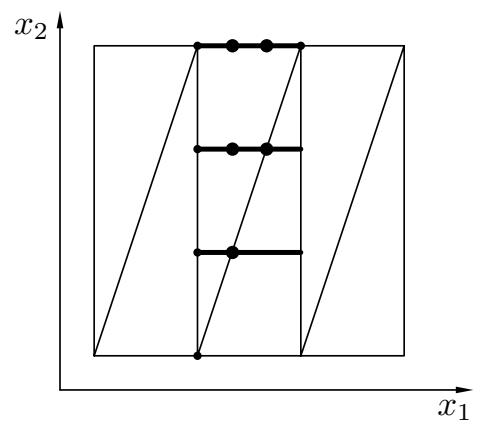

(a) Points where $\sigma_{i}$ is uniquely determined.

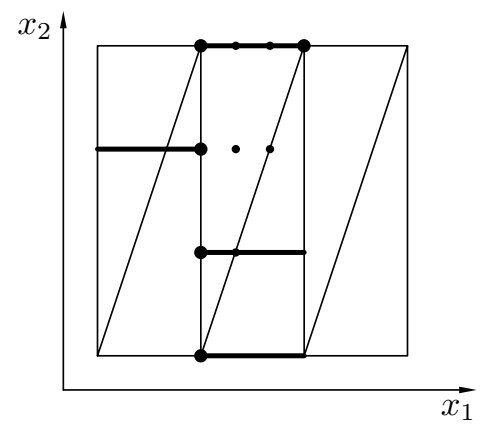

(b) Points where $\sigma_{i}$ can be chosen (here one choice).

Figure 5. Choice of $\sigma_{i}$ in dependence of $X_{i}$ in the case of operator $\mathrm{S}_{h}, k=3$.

Since the definition of the $\sigma_{i}$ is different from that of $\mathrm{Z}_{h}$ in Section 3 we will clarify this here: $\sigma_{i}$ is (not necessarily uniquely) determined according to the following three properties, compare Figure 5.

(P1) $\sigma_{i}$ is parallel to the $x_{1}$-axis $/ x_{1}, x_{2}$-plane.

(P2) $X_{i} \in \overline{\sigma_{i}}$.

(P3) There exists an edge/face $\varsigma$ of some element $e$ such that the projection of $\varsigma$ on the $x_{1}$-axis $/ x_{1}, x_{2}$-plane is identical with the projection of $\sigma_{i}$.

In connection with (P3) we have to note that $\sigma_{i}$ is not necessary an edge/face of one element, see also Figure 5. Nevertheless, $\sigma_{i}$ together with $\mathcal{P}_{k}^{d-1}$ or $\mathcal{Q}_{k}^{d-1}$ is a Lagrangian finite element of dimension $d-1$, which follows from the tensor-product character of the elements $e$. For simplicity, we will use the terminology " $\sigma_{i}$ is an edge/face". We remark in particular that in the case of simplicial elements and $k \geq 2$ there is no $d$-dimensional finite element $e^{\prime} \subset S_{e}$ such that $\sigma_{i} \subset \overline{e^{\prime}}$. This implies that $\mathcal{P}_{k, \sigma_{i}} \neq\left. V_{h}\right|_{\sigma_{i}}$ and in general $\Pi_{\sigma_{i}} v_{h} \neq\left. v_{h}\right|_{\sigma_{i}}$ for $v_{h} \in V_{h}$. That means that we lose Property 2 in Section 1 . However, we need in the proofs only $\Pi_{\sigma_{i}} w=w$ for $w \in \mathcal{P}_{k, \sigma_{i}}$ which is of course satisfied.

Because $\sigma_{i}$ is said to be a small edge/face this implies

$$
h_{j} \leq h_{d} \quad \text { in } S_{e} \quad(j=1, \ldots, d)
$$

Note that in three dimensions and according to $(2.2,2.3)$, only elements with $h_{1} \sim h_{2} \lesssim h_{3}$ can be treated. But this is sufficient to handle edge singularities, see Section 7.

We will see that for the operator $\mathrm{S}_{h}$ anisotropic estimates of type $(m, \ell), m<\ell \leq k+1$, can be derived. The main difficulty is to prove the stability estimate. The approximation property follows then easily using Lemma 1 from Section 2. To elucidate the different techniques for derivatives in $x_{1}$ - and $x_{d}$-direction we first formulate and prove two lemmata. Then we establish the main theorem of this section. Finally, we give an example which shows that estimates of type $(m, m), 1 \leq m \leq k+1$, are impossible.

Lemma 4. Consider an element $e$ of a mesh of tensor product type and assume that (4.1) is valid. Then the derivative of $\mathrm{S}_{h} u$ in $x_{d}$-direction satisfies an $(1,1)$-estimate. The relation

$$
\left\|\frac{\partial}{\partial x_{d}} \mathrm{~S}_{h} u ; L^{q}(e)\right\| \lesssim(\operatorname{meas} e)^{1 / q-1 / p}\left|u ; W^{1, p}\left(S_{e}\right)\right|
$$

holds for $u \in W^{1, p}\left(S_{e}\right)$ and all $p, q \in[1, \infty]$. 
Proof. Using the definition of the operator $\mathrm{S}_{h}$ (in analogy to (3.4)), the Hölder inequality, estimate (3.11), and the trace Theorem (3.13), we obtain for all $w \in \mathcal{P}_{0}^{d}$

$$
\begin{aligned}
\left\|\frac{\partial}{\partial x_{d}} \mathrm{~S}_{h} u ; L^{q}(e)\right\| & =\left\|\frac{\partial}{\partial x_{d}} \mathrm{~S}_{h}(u-w) ; L^{q}(e)\right\| \leq \sum_{i \in I_{e}}\left\|\frac{\partial \varphi_{i}}{\partial x_{d}} ; L^{q}(e)\right\|\left|\int_{\sigma_{i}}(u-w) \psi_{i}\right| \\
& \lesssim h_{d}^{-1}(\operatorname{meas} e)^{1 / q} \sum_{i \in I_{e}}\left\|u-w ; L^{1}\left(\sigma_{i}\right)\right\|\left\|\psi_{i} ; L^{\infty}\left(\sigma_{i}\right)\right\| \\
& \lesssim h_{d}^{-1}(\operatorname{meas} e)^{1 / q} \sum_{i \in I_{e}}\left(\operatorname{meas} \sigma_{i}\right)(\operatorname{meas} e)^{-1 / p} \sum_{|\alpha| \leq 1} h^{\alpha}\left\|D^{\alpha}(u-w) ; L^{p}\left(S_{e}\right)\right\|\left(\operatorname{meas} \sigma_{i}\right)^{-1} \\
& \lesssim h_{d}^{-1}(\operatorname{meas} e)^{1 / q-1 / p} \sum_{|\alpha| \leq 1} h^{\alpha}\left\|D^{\alpha}(u-w) ; L^{p}\left(S_{e}\right)\right\| .
\end{aligned}
$$

Using Lemma 1 with $m=0, \ell=1$, and relying on (4.1) we obtain the assertion.

Lemma 5. Consider an element e of a mesh of tensor product type and assume that (4.1) is valid. Then the derivative of $\mathrm{S}_{h} u$ in $x_{1}$-direction satisfies an $(1,2)$-estimate. The relation

$$
\left\|\frac{\partial}{\partial x_{1}} \mathrm{~S}_{h} u ; L^{q}(e)\right\| \lesssim(\operatorname{meas} e)^{1 / q-1 / p} \sum_{|\alpha| \leq 1} h^{\alpha}\left|D^{\alpha} u ; W^{1, p}\left(S_{e}\right)\right|
$$

holds for $u \in W^{2, p}\left(S_{e}\right)$ and all $p, q \in[1, \infty]$.

Proof. Let $w=w\left(x_{d}\right) \in \mathcal{P}_{k}^{1}$. Then we get in analogy to the proof of Lemma 4

$$
\left\|\frac{\partial}{\partial x_{1}} \mathrm{~S}_{h} u ; L^{q}(e)\right\| \lesssim h_{1}^{-1}(\operatorname{meas} e)^{1 / q} \sum_{i \in I_{e}}\left(\operatorname{meas} \sigma_{i}\right)^{-1}\left\|u-w ; L^{1}\left(\sigma_{i}\right)\right\| .
$$

Denote by $\sigma$ the smallest of the domains $\sigma_{i}, i \in I_{e}$. Introduce now $k+1$ (simply connected) $(d-1)$-dimensional domains $\zeta_{j} \subset S_{e}$ such that for all $\sigma_{i}\left(i \in I_{e}\right)$ there exists a $\zeta_{j} \supset \sigma_{i}$. Note that, due to $(2.5), \zeta_{j}(j=0, \ldots, k)$ is isotropic with a diameter of order $h_{1}$, and therefore meas $\sigma_{i} \sim$ meas $\zeta_{j} \sim$ meas $\sigma$ for all $i$ and $j$. Consequently, we obtain

$$
\begin{aligned}
\left\|\frac{\partial}{\partial x_{1}} \mathrm{~S}_{h} u ; L^{q}(e)\right\| & \lesssim h_{1}^{-1}(\operatorname{meas} e)^{1 / q}(\operatorname{meas} \sigma)^{-1} \sum_{j=0}^{k}\left\|u-w ; L^{1}\left(\zeta_{j}\right)\right\| \\
& \leq h_{1}^{-1}(\operatorname{meas} e)^{1 / q}(\operatorname{meas} \sigma)^{-1} \sum_{j=0}^{k} \sum_{\substack{|\alpha| \leq 1 \\
\alpha_{d}=0}} h^{\alpha}\left\|D^{\alpha}(u-w) ; L^{1}\left(\zeta_{j}\right)\right\| .
\end{aligned}
$$

Observe now that $w=w_{j}=$ const. on $\zeta_{j}$. On the other hand, because the $\zeta_{j}$ have different $x_{d}$-coordinate, we can define $w$ from given $w_{j}(j=0, \ldots, k)$. So we can use Lemma 1 for dimension $d-1$ to choose $w_{j} \in \mathcal{P}_{0}^{d-1}$ such that

$$
\sum_{\substack{|\alpha| \leq 1 \\ \alpha_{d}=0}} h^{\alpha}\left\|D^{\alpha}\left(u-w_{j}\right) ; L^{1}\left(\zeta_{j}\right)\right\| \lesssim \sum_{\substack{|\alpha|=1 \\ \alpha_{d}=0}} h^{\alpha}\left\|D^{\alpha} u ; L^{1}\left(\zeta_{j}\right)\right\|
$$


and to conclude with the trace Theorem (3.13) (applied for each $\zeta_{j}$ )

$$
\begin{aligned}
\left\|\frac{\partial}{\partial x_{1}} \mathrm{~S}_{h} u ; L^{q}(e)\right\| & \lesssim(\operatorname{meas} e)^{1 / q}(\operatorname{meas} \sigma)^{-1} \sum_{j=0}^{k} \sum_{\substack{|\alpha|=1 \\
\alpha_{d}=0}}\left\|D^{\alpha} u ; L^{1}\left(\zeta_{j}\right)\right\| \\
& \lesssim(\operatorname{meas} e)^{1 / q-1 / p} \sum_{\substack{|\alpha|=1 \\
\alpha_{d}=0}} \sum_{\substack{|\beta| \leq 1 \\
\alpha^{\beta}}} h^{\beta}\left\|D^{\alpha+\beta} u ; L^{p}\left(S_{e}\right)\right\| .
\end{aligned}
$$

Thus the proposition is proved.

By analogy we can treat the derivative with respect to $x_{2}$ in the three-dimensional case.

Theorem 6. Assume that (4.1) is valid. Then the modified Scott-Zhang operator $\mathrm{S}_{h}$ satisfies on anisotropic meshes of tensor-product type the following estimates of type $(m, \ell)$ :

$$
\begin{aligned}
\left|\mathrm{S}_{h} u ; W^{m, q}(e)\right| & \lesssim(\text { meas } e)^{1 / q-1 / p} \sum_{|\alpha| \leq \ell-m} h^{\alpha}\left|D^{\alpha} u ; W^{m, p}\left(S_{e}\right)\right|, \\
\left|u-\mathrm{S}_{h} u ; W^{m, q}(e)\right| & \lesssim(\text { meas } e)^{1 / q-1 / p} \sum_{|\alpha|=\ell-m} h^{\alpha}\left|D^{\alpha} u ; W^{m, p}\left(S_{e}\right)\right|,
\end{aligned}
$$

$0 \leq m \leq \ell-1 \leq k$, provided that $u \in W^{\ell, p}\left(S_{e}\right)$. For (4.5) the numbers $p, q \in[1, \infty]$ must be such that $W^{\bar{\ell}, p}(e) \hookrightarrow W^{m, q}(e)$. For $m \geq 2$ we exclude triangular and tetrahedral elements.

Proof. Consider first the stability estimate (4.4). For $m=0$, (4.4) can be proved as (3.9). For $m=1,(4.4)$ is proved in Lemmata 4 and 5. Let $m \geq 2$. Consider a multi-index $\gamma$ with $|\gamma|=m$ and define $m_{2}:=\gamma_{d}$, $m_{1}=m-m_{2}$. For arbitrary $\omega_{1}=\omega_{1,1}\left(x_{1}, \ldots, x_{d-1}\right) \omega_{1,2}\left(x_{d}\right), \omega_{1,1} \in \mathcal{P}_{m_{1}-1}^{d-1}, \omega_{1,2} \in \mathcal{P}_{k}^{1}$, (that is why we exclude simplicial elements) and $\omega_{2} \in \mathcal{P}_{m-1}^{d}$ we obtain in analogy to the proof of Lemma 5

$$
\begin{aligned}
\left\|D^{\gamma} \mathrm{S}_{h} u ; L^{q}(e)\right\| & =\left\|D^{\gamma} \mathrm{S}_{h}\left(\left(u-\omega_{2}\right)-\omega_{1}\right) ; L^{q}(e)\right\| \\
& \lesssim h^{-\gamma}(\operatorname{meas} e)^{1 / q}(\operatorname{meas} \sigma)^{-1} \sum_{i \in I_{e}}\left\|u-\omega_{2}-\omega_{1} ; L^{1}\left(\sigma_{i}\right)\right\| \\
& \lesssim h^{-\gamma}(\operatorname{meas} e)^{1 / q}(\operatorname{meas} \sigma)^{-1} \sum_{j=0}^{k} \sum_{\substack{|\alpha| \leq m_{1} \\
\alpha_{d}=0}} h^{\alpha}\left\|D^{\alpha}\left(u-\omega_{2}-\omega_{1}\right) ; L^{1}\left(\zeta_{j}\right)\right\| .
\end{aligned}
$$

Then we determine $w_{j} \in \mathcal{P}_{m_{1}-1}^{d-1}(j=0, \ldots, k)$ such that

$$
\sum_{\substack{|\alpha| \leq m_{1} \\ \alpha_{d}=0}} h^{\alpha}\left\|D^{\alpha}\left(u-\omega_{2}-w_{j}\right) ; L^{1}\left(\zeta_{j}\right)\right\| \lesssim \sum_{\substack{|\alpha|=m_{1} \\ \alpha_{d}=0}} h^{\alpha}\left\|D^{\alpha}\left(u-\omega_{2}\right) ; L^{1}\left(\zeta_{j}\right)\right\| .
$$

Note that the $w_{j}$ depend on $\left(u-\omega_{2}\right)$ and $\omega_{2}$ is still to be chosen. The polynomial $\omega_{1}$ is now determined by the $w_{j}(j=0, \ldots, k)$ such that the estimate can be continued by

$$
\left\|D^{\gamma} \mathrm{S}_{h} u ; L^{q}(e)\right\| \lesssim h_{d}^{-m_{2}}(\operatorname{meas} e)^{1 / q}(\operatorname{meas} \sigma)^{-1} \sum_{j=0}^{k} \sum_{\substack{|\alpha|=m_{1} \\ \alpha_{d}=0}}\left\|D^{\alpha}\left(u-\omega_{2}\right) ; L^{1}\left(\zeta_{j}\right)\right\| .
$$


Thus the factor $h_{1}^{-m_{1}}$ is eliminated. We proceed now as in the proof of Lemma 4. Using the trace Theorem (3.13) for all $j, \alpha$ and with $\ell-m_{1} \geq \ell-m \geq 1$ instead of $\ell$ we conclude

$$
\begin{aligned}
\left\|D^{\gamma} \mathrm{S}_{h} u ; L^{q}(e)\right\| & \lesssim h_{d}^{-m_{2}}(\text { meas } e)^{1 / q-1 / p} \sum_{\substack{|\alpha|=m_{1} \\
\alpha_{d}=0}} \sum_{|\beta| \leq \ell-m_{1}} h^{\beta}\left\|D^{\alpha+\beta}\left(u-\omega_{2}\right) ; L^{p}\left(S_{e}\right)\right\| \\
& \lesssim h_{d}^{-m_{2}}(\text { meas } e)^{1 / q-1 / p} \sum_{|\delta| \leq \ell-m|\beta| \leq m_{2}} \sum^{\beta+\delta}\left|D^{\beta+\delta}\left(u-\omega_{2}\right) ; W^{m_{1}, p}\left(S_{e}\right)\right| .
\end{aligned}
$$

Using Corollary 2 (Section 2) we obtain

$$
\begin{aligned}
\left\|D^{\gamma} \mathrm{S}_{h} u ; L^{q}(e)\right\| & \lesssim h_{d}^{-m_{2}}(\text { meas } e)^{1 / q-1 / p} \sum_{|\delta| \leq \ell-m} \sum_{|\beta|=m_{2}} h^{\beta+\delta}\left|D^{\beta+\delta} u ; W^{m_{1}, p}\left(S_{e}\right)\right| \\
& \lesssim(\text { meas } e)^{1 / q-1 / p} \sum_{|\delta| \leq \ell-m} h^{\delta}\left|D^{\delta} u ; W^{m, p}\left(S_{e}\right)\right| .
\end{aligned}
$$

Here we used $h^{\beta} \leq h_{d}^{m_{2}}$ for $|\beta|=m_{2}$ which follows from (4.1). Thus (4.4) is proved.

For proving estimate (4.5) we need (4.4) and the assumptions on $p$ and $q$. Since these parameters were chosen such that $W^{\ell, p}(e) \hookrightarrow W^{m, q}(e)$, we have also $W^{\ell-m, p}(e) \hookrightarrow L^{q}(e)$, this means

$$
\left\|v ; L^{q}(e)\right\| \lesssim(\text { meas } e)^{1 / q-1 / p} \sum_{|\alpha| \leq \ell-m} h^{\alpha}\left\|D^{\alpha} v ; L^{p}(e)\right\|
$$

for all $v \in W^{\ell-m, p}(e)$. Applying this estimate for all derivatives $D^{\alpha}$ with $|\alpha|=m$ and summing up the resulting inequalities, we obtain for $v \in W^{\ell, p}(e)$

$$
\left|v ; W^{m, q}(e)\right| \lesssim(\text { meas } e)^{1 / q-1 / p} \sum_{|\alpha| \leq \ell-m} h^{\alpha}\left|D^{\alpha} v ; W^{m, p}(e)\right| .
$$

Together with (4.4) we conclude that for all $w \in \mathcal{P}_{\ell-1}^{d}$ the following estimate holds,

$$
\begin{aligned}
\left|u-\mathrm{S}_{h} u ; W^{m, q}(e)\right| & \leq\left|u-w ; W^{m, q}(e)\right|+\left|\mathrm{S}_{h}(u-w) ; W^{m, q}(e)\right| \\
& \lesssim(\operatorname{meas} e)^{1 / q-1 / p} \sum_{|\alpha| \leq \ell-m} h^{\alpha}\left|D^{\alpha}(u-w) ; W^{m, p}\left(S_{e}\right)\right| .
\end{aligned}
$$

With Lemma 1 the proposition is proved.

Finally, we want to give an example which shows that

$$
\left|\mathrm{S}_{h} u ; W^{1,2}(e)\right| \lesssim\left\|u ; W^{1,2}\left(S_{e}\right)\right\|
$$

does not hold for general $u \in W^{1,2}\left(S_{e}\right)$.

Example 4. Consider $k=1$ and a triangle with the vertices $X_{1}=(0,0), X_{2}=(h, 0)$, and $X_{3}=(0,1)$, and let $\sigma_{1}=(-h, 0) \times\{0\}, \sigma_{2}=(0, h) \times\{0\}$, compare Figure 6. For $u=r^{\varepsilon} \sin (\theta / 2)(r, \theta$ are here polar coordinates $)$ we obtain

$$
\begin{aligned}
\left.u\right|_{\sigma_{1}}=\left|x_{1}\right|^{\varepsilon} & \Rightarrow \quad\left(\Pi_{\sigma_{1}} u\right)\left(X_{1}\right)=\int_{0}^{h} x^{\varepsilon}\left(-\frac{6 x}{h^{2}}+\frac{4}{h}\right) \sim h^{\varepsilon} \\
\left.u\right|_{\sigma_{2}}=0 & \Rightarrow \quad\left(\Pi_{\sigma_{2}} u\right)\left(X_{2}\right)=0
\end{aligned}
$$




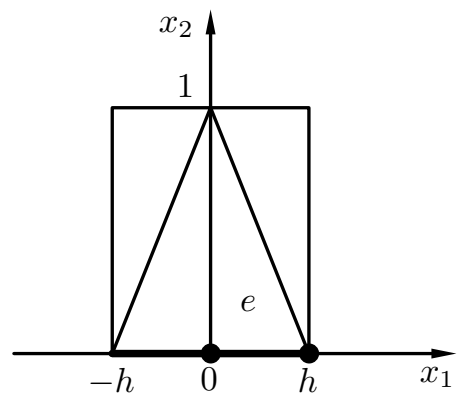

FiguRE 6. Illustration of Example 4.

Consequently,

$$
\frac{\partial \mathrm{S}_{h} u}{\partial x_{1}} \sim h^{\varepsilon-1}, \quad\left|\mathrm{~S}_{h} u ; W^{1,2}(e)\right| \gtrsim h^{\varepsilon-1}(\operatorname{meas} e)^{1 / 2}=h^{\varepsilon-1 / 2} \rightarrow \infty
$$

for $h \rightarrow 0, \varepsilon<1 / 2$. But

$$
\left|u ; W^{1,2}\left(S_{e}\right)\right|^{2} \lesssim \int_{0}^{1} \int_{0}^{\pi}\left(r^{\varepsilon-1} \sin \frac{\theta}{2}\right)^{2} r \mathrm{~d} \theta \mathrm{d} r \sim \int_{0}^{1} r^{2(\varepsilon-1)+1} \mathrm{~d} r<\infty
$$

for $\varepsilon>0$. Thus (4.8) does not hold.

\subsection{Stability in weighted Sobolev spaces}

We have seen in Example 4 that $\mathrm{S}_{h} u$ does not satisfy an estimate of type $(1,1)$. However, $\mathrm{S}_{h}$ can be applied in some situations where $u \notin W^{2, p}\left(S_{e}\right)$ for some $p$ we are interested in.

We restrict ourselves to the three-dimensional case, consider an arbitrary bounded domain $G \subset \mathbb{R}^{3}$ with zero distance to the $x_{3}$-axis (the $x_{3}$-axis may intersect $G$ but this is not typical), and introduce cylindrical coordinates via $x_{1}=r \cos \theta, x_{2}=r \sin \theta$. Define for $\ell \in \mathbb{N}_{0}, p \in[1, \infty], \beta \in \mathbb{R}$, the weighted Sobolev space

$$
\begin{aligned}
V_{\beta}^{\ell, p}(G) & :=\left\{v \in \mathcal{D}^{\prime}(G):\left\|v ; V_{\beta}^{\ell, p}(G)\right\|<\infty\right\} \\
\left\|v ; V_{\beta}^{\ell, p}(G)\right\|^{p} & :=\sum_{|\alpha| \leq \ell} \int_{G}\left|r^{\beta-\ell+|\alpha|} D^{\alpha} v\right|^{p} .
\end{aligned}
$$

Such spaces are relevant in the treatment of singular functions of the type $v=r^{\lambda} \sin \lambda \theta$ or $v=r^{\lambda} \cos \lambda \theta$, $\lambda \in(0,1)$. Notice that

$$
\begin{array}{ll}
v \in W^{s, 2}(G) & \Longleftrightarrow s<1+\lambda, \\
v \in V_{\beta}^{s, 2}(G) \quad \forall s \geq 0 \quad \Longleftrightarrow \beta>s-1-\lambda .
\end{array}
$$

For our application in Section 7 we need the stability of the modified Scott-Zhang operator in these weighted spaces.

Lemma 7. Consider an element e of a mesh of tensor product type and assume that (4.1) is valid. Let $m$ be an integer and $\beta, p, q$ be real numbers with $0 \leq m \leq k, \beta<2-2 / p, \beta \leq 1, p, q \in[1, \infty]$, and assume that the $x_{3}$-axis proceeds through $S_{e}$. Then for $u \in W^{m, p}\left(S_{e}\right) \cap V_{\beta}^{m+1, p}\left(S_{e}\right)$ the stability estimate

$$
\left|\mathrm{S}_{h} u ; W^{m, q}(e)\right| \lesssim(\operatorname{meas} e)^{1 / q-1 / p} h_{1}^{-\beta} \sum_{|\alpha|=m-1} \sum_{|t|=1} h^{t}\left\|D^{\alpha+t} u ; V_{\beta}^{1, p}\left(S_{e}\right)\right\|
$$


holds. For $m \geq 2$ we exclude tetrahedral elements.

Proof. We start with estimate (4.6) which was obtained in the proof of Theorem 6. Let $\gamma$ be a multi-index with $|\gamma|=m, m_{1}=m-\gamma_{3}$, and $\omega_{2} \in \mathcal{P}_{m-1}^{d}$. Then there holds

$$
\left\|D^{\gamma} \mathrm{S}_{h} u ; L^{q}(e)\right\| \lesssim h_{3}^{-\gamma_{3}}(\operatorname{meas} e)^{1 / q}(\operatorname{meas} \sigma)^{-1} \sum_{j=0}^{k} \sum_{\substack{\alpha \mid=m-\gamma_{3} \\ \alpha_{3}=0}}\left\|D^{\alpha}\left(u-\omega_{2}\right) ; L^{1}\left(\zeta_{j}\right)\right\| .
$$

Let $\gamma_{3}>0$, then we can continue, similar to the proof of Theorem 6 , with the trace theorem because we assumed $u \in W^{m, p}\left(S_{e}\right)$.

$$
\left\|D^{\gamma} \mathrm{S}_{h} u ; L^{q}(e)\right\| \lesssim h_{3}^{-\gamma_{3}}(\text { meas } e)^{1 / q-1 / p} \sum_{\substack{|\alpha|=m-\gamma_{3} \\ \alpha_{3}=0}} \sum_{|\delta| \leq \gamma_{3}} h^{\delta}\left\|D^{\alpha+\delta}\left(u-\omega_{2}\right) ; L^{p}\left(S_{e}\right)\right\| .
$$

Using Corollary 2 we obtain

$$
\begin{aligned}
\left\|D^{\gamma} \mathrm{S}_{h} u ; L^{q}(e)\right\| & \lesssim h_{3}^{-\gamma_{3}}(\text { meas } e)^{1 / q-1 / p} \sum_{\substack{|\alpha|=m-\gamma_{3} \\
\alpha_{3}=0}} \sum_{|\delta|=\gamma_{3}} h^{\delta}\left\|D^{\alpha+\delta} u ; L^{p}\left(S_{e}\right)\right\| \\
& \lesssim(\text { meas } e)^{1 / q-1 / p} \sum_{|\alpha|=m}\left\|D^{\alpha} u ; L^{p}\left(S_{e}\right)\right\| .
\end{aligned}
$$

We estimate the right-hand side via the trivial embeddings $V_{\beta}^{1, p}\left(S_{e}\right) \hookrightarrow V_{\beta-1}^{0, p}\left(S_{e}\right) \hookrightarrow L^{p}\left(S_{e}\right), \beta \leq 1$, which leads with (4.1) to

$$
\begin{aligned}
\sum_{|\alpha|=m}\left\|D^{\alpha} u ; L^{p}\left(S_{e}\right)\right\| & \sim \sum_{|\alpha|=m-1} \sum_{|t|=1}\left\|D^{\alpha+t} u ; L^{p}\left(S_{e}\right)\right\| \\
& \lesssim h_{1}^{-\beta+1} \sum_{|\alpha|=m-1} \sum_{|t|=1}\left\|r^{\beta-1} D^{\alpha+t} u ; L^{p}\left(S_{e}\right)\right\| \\
& \lesssim h_{1}^{-\beta} \sum_{|\alpha|=m-1} \sum_{|t|=1} h^{t}\left\|D^{\alpha+t} u ; V_{\beta}^{1, p}\left(S_{e}\right)\right\|,
\end{aligned}
$$

which is the desired result.

For $\gamma_{3}=0$ we use (4.12) with $\omega_{2}=0$ and estimate the $L^{1}\left(\zeta_{j}\right)$-norms against weighted norms via the Hölder inequality:

$$
\left\|v ; L^{1}\left(\zeta_{j}\right)\right\| \leq\left\|r^{-\beta} ; L^{p^{\prime}}\left(\zeta_{j}\right)\right\| \cdot\left\|r^{\beta} v ; L^{p}\left(\zeta_{j}\right)\right\|
$$

with $p^{\prime}$ from $1 / p+1 / p^{\prime}=1$. The $L^{p^{\prime}}\left(\zeta_{j}\right)$-norm of $r^{-\beta}$ is finite if and only if $p^{\prime} \beta<2$ which is equivalent to $\beta<2-2 / p$. Using meas $\sigma \sim \operatorname{meas} \zeta_{j} \sim h_{1}^{2}$ for all $j$, and $r \lesssim h_{1}$ we get

$$
\left\|r^{-\beta} ; L^{p^{\prime}}\left(\zeta_{j}\right)\right\| \lesssim h_{1}^{\left(-\beta p^{\prime}+2\right) / p^{\prime}} \sim(\text { meas } \sigma)^{1-1 / p} h_{1}^{-\beta}
$$

The application of $W^{1, p}\left(S_{e}\right) \hookrightarrow L^{p}\left(\zeta_{j}\right)$ to $r^{\beta} v$ implies the trace theorem $V_{\beta}^{1, p}\left(S_{e}\right) \hookrightarrow V_{\beta}^{0, p}\left(\zeta_{j}\right)$ which leads to

$$
\left\|r^{\beta} v ; L^{p}\left(\zeta_{j}\right)\right\| \lesssim(\text { meas } \sigma)^{1 / p}(\text { meas } e)^{-1 / p} \sum_{|s| \leq 1} h_{1}^{1-|s|} h^{s}\left\|r^{\beta-1+|s|} D^{s} v ; L^{p}\left(S_{e}\right)\right\|
$$


Combining these estimates we obtain

$$
\left\|v ; L^{1}\left(\zeta_{j}\right)\right\| \leq \operatorname{meas} \sigma(\text { meas } e)^{-1 / p} h_{1}^{-\beta} \sum_{|s| \leq 1} h_{1}^{1-|s|} h^{s}\left\|r^{\beta-1+|s|} D^{s} v ; L^{p}\left(S_{e}\right)\right\|
$$

and thus with (4.12)

$$
\begin{aligned}
\left\|D^{\gamma} \mathrm{S}_{h} u ; L^{q}(e)\right\| & \lesssim(\text { meas } e)^{1 / q}(\operatorname{meas} \sigma)^{-1} \sum_{j=0}^{k} \sum_{|\alpha|=m}\left\|D^{\alpha} u ; L^{1}\left(\zeta_{j}\right)\right\| \\
& \lesssim(\text { meas } e)^{1 / q-1 / p} h_{1}^{-\beta} \sum_{|\alpha|=m} \sum_{|s| \leq 1} h_{1}^{1-|s|} h^{s}\left\|r^{\beta-1+|s|} D^{\alpha+s} u ; L^{p}\left(S_{e}\right)\right\| .
\end{aligned}
$$

The last step to derive (4.11) is done by a rearrangement of the terms at the right-hand side, namely

$$
\begin{aligned}
\sum_{|t|=1} \sum_{|s| \leq 1} h_{1}^{1-|s|} h^{s}\left\|r^{\beta-1+|s|} D^{t+s} u ; L^{p}\left(S_{e}\right)\right\| & =\sum_{|t|=1} \sum_{|s|=1} h^{s}\left\|r^{\beta} D^{t+s} u ; L^{p}\left(S_{e}\right)\right\|+\sum_{|t|=1} h_{1}\left\|r^{\beta-1} D^{t} u ; L^{p}\left(S_{e}\right)\right\| \\
& \lesssim \sum_{|t|=1} \sum_{|s|=1} h^{s}\left\|r^{\beta} D^{t+s} u ; L^{p}\left(S_{e}\right)\right\|+\sum_{|s|=1} h^{s}\left\|r^{\beta-1} D^{s} u ; L^{p}\left(S_{e}\right)\right\| \\
& \sim \sum_{|s|=1} h^{s}\left\|D^{s} u ; V_{\beta}^{1, p}\left(S_{e}\right)\right\| .
\end{aligned}
$$

Together with (4.17) we conclude (4.11) in the case $\gamma_{3}=0$.

\section{The operator $\mathrm{L}_{h}$ : A MOdification of $\mathrm{Z}_{h}$ By ChOOSING LARGE Sides WITH A PROJECTION PROPERTY}

In contrast to Section 4 we will now employ large edges/faces and investigate the resulting operator $\mathrm{L}_{h}$. We still assume that $e$ is an element of tensor product type, see Definition 1 in Section 2. The notation is used as follows: We keep Properties (P1, P2, P3) from Section 4 and simply turn the relation (4.1):

$$
h_{j} \geq h_{d} \quad \text { in } S_{e} \quad(j=1, \ldots, d) .
$$

But due to the conclusions of Example 1 in Section 3, we do not have so much freedom for the choice of the $\sigma_{i}$ as in the case of $\mathrm{S}_{h}$. We must assume the following projection property (P4), compare also Figure 7 .

(P4) If the projections of any two points $X_{i}$ and $X_{j}$ on the $x_{1}$-axis $/ x_{1}, x_{2}$-plane coincide then so do the projections of $\sigma_{i}$ and $\sigma_{j}$.

We can prove the results of Theorem 6 for this case as well. Moreover, these results extend to the case $m=\ell$. But in contrast to the needle elements of Section 4 the three-dimensional elements are now flat, $h_{1} \sim h_{2} \gtrsim h_{3}$. The idea for this choice of $\sigma_{i}$ was found in Chapter 5 of [15] where the special case of rectangular and brick elements was considered for $k=1, p=q=2$. We extend this theory to more element types and to general $k \in \mathbb{N}, p, q \in[1, \infty]$. Our proof differs from that in [15].

We start as in Section 4 with the separate consideration of the stability of first derivatives of $\mathrm{L}_{h} u$. This time the derivative in $x_{1}$-direction is the simpler one. 


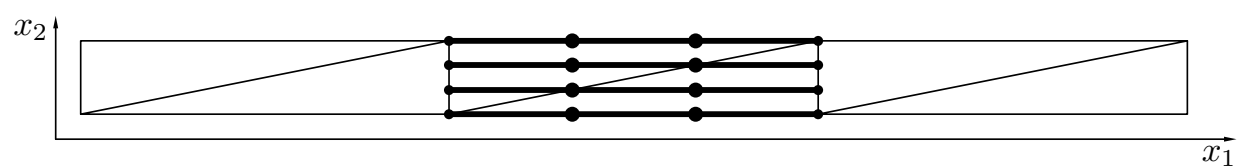

(a) Points where $\sigma_{i}$ is uniquely determined.

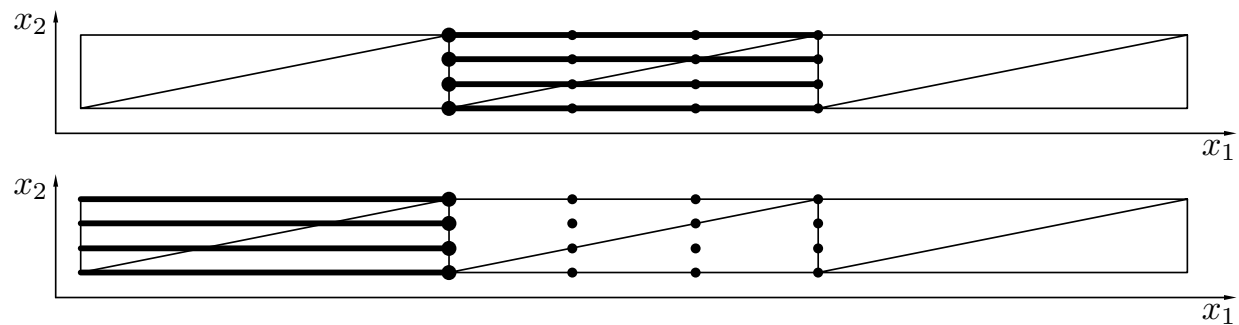

(b) Two choices for $\sigma_{i}$ for points on vertical mesh lines.

Figure 7. Choice of $\sigma_{i}$ in dependence of $X_{i}$ in the case of operator $\mathrm{L}_{h}$.

Lemma 8. Consider an element e of a mesh of tensor product type and assume that (5.1) is valid. Then the estimate of type $(1,1)$

$$
\left\|\frac{\partial}{\partial x_{n}} \mathrm{~L}_{h} u ; L^{q}(e)\right\| \lesssim(\text { meas } e)^{1 / q-1 / p}\left|u ; W^{1, p}\left(S_{e}\right)\right|, \quad n=1, \ldots, d
$$

holds for $u \in W^{1, p}\left(S_{e}\right)$ and all $p, q \in[1, \infty]$.

Proof. For $n=1, \ldots, d-1$ the proof can be carried out with the same arguments as the proof of Lemma 4 . The only difference is that the role of $x_{d}$ and $h_{d}$ is now played by $x_{n}$ and $h_{n}$.

For the case $n=d$ we will reformulate $\mathrm{L}_{h} u$. For this consider first a one-dimensional situation, that means a single finite element formed by an interval $(\xi, \eta)$. Let $\phi_{i}, i=0, \ldots, k$, be the nodal basis functions in $(\xi, \eta)$. We change now to a new basis

$$
\chi_{i}=\sum_{j=0}^{i} \phi_{j}, \quad i=0, \ldots, k
$$

Consequently,

$$
\sum_{i=0}^{k} a_{i} \phi_{i}=\sum_{i=0}^{k-1}\left(a_{i}-a_{i+1}\right) \chi_{i}+a_{k}
$$

where we also used that $\sum_{i=0}^{k} \phi_{i}=1$. Note further that

$$
\left\|\chi_{i} ; L^{\infty}(\xi, \eta)\right\| \lesssim 1, \quad\left\|\chi_{i}^{\prime} ; L^{\infty}(\xi, \eta)\right\| \lesssim|\eta-\xi|^{-1} .
$$

We use this kind of a new basis in the case of a rectangular element $e=\left(\xi_{1}, \eta_{1}\right) \times\left(\xi_{2}, \eta_{2}\right)$. The nodal basis functions are (for simplicity with a double index)

$$
\varphi_{i, j}\left(x_{1}, x_{2}\right)=\phi^{i}\left(x_{1}\right) \phi_{j}\left(x_{2}\right), \quad i, j=0, \ldots, k,
$$


where $\phi^{i}$ and $\phi_{j}$ are the nodal basis functions with respect to $\left(\xi_{1}, \eta_{1}\right)$ and $\left(\xi_{2}, \eta_{2}\right)$, respectively. Thus

$$
\begin{aligned}
\mathrm{L}_{h} u & =\sum_{i=0}^{k} \sum_{j=0}^{k} a_{i, j} \phi^{i}\left(x_{1}\right) \phi_{j}\left(x_{2}\right) \\
& =\sum_{i=0}^{k} \phi^{i}\left(x_{1}\right)\left(\sum_{j=0}^{k-1}\left(a_{i, j}-a_{i, j+1}\right) \chi_{j}\left(x_{2}\right)+a_{i, k}\right), \\
\frac{\partial}{\partial x_{2}} \mathrm{~L}_{h} u & =\sum_{i=0}^{k} \phi^{i}\left(x_{1}\right) \sum_{j=0}^{k-1}\left(a_{i, j}-a_{i, j+1}\right) \chi_{j}^{\prime}\left(x_{2}\right) .
\end{aligned}
$$

Because of Property (P4) the subdomains $\sigma_{i, j}$ belonging to the node $(i, j)$ depend only on $i$. We can write

$$
\begin{aligned}
a_{i, j} & =\int_{\sigma_{i, j}} \psi_{i}\left(x_{1}\right) u\left(x_{1}, y_{j}\right) \mathrm{d} x_{1}, \\
a_{i, j}-a_{i, j+1} & =-\int_{\sigma_{i, j}} \psi_{i}\left(x_{1}\right) \int_{y_{j}}^{y_{j}+1} \frac{\partial u}{\partial x_{2}}\left(x_{1}, y\right) \mathrm{d} y \mathrm{~d} x_{1}, \\
\sum_{j=0}^{k-1}\left|a_{i, j}-a_{i, j+1}\right| & \leq \int_{S_{e}}\left|\psi_{i} \frac{\partial u}{\partial x_{2}}\right|,
\end{aligned}
$$

where $y_{j}$ is the value of the $x_{2}$-coordinate of points $X_{i, j}$. The proof of (5.2) is now standard:

$$
\begin{aligned}
\left\|\frac{\partial}{\partial x_{d}} \mathrm{~L}_{h} u ; L^{q}(e)\right\| & \lesssim \sum_{i=0}^{k} \sum_{j=0}^{k-1}\left|a_{i, j}-a_{i, j+1}\right| \cdot\left\|\phi^{i}\left(x_{1}\right) \chi_{j}^{\prime}\left(x_{2}\right) ; L^{q}(e)\right\| \\
& \lesssim h_{2}^{-1}(\operatorname{meas} e)^{1 / q} \sum_{i=0}^{k} \int_{S_{e}}\left|\psi_{i} \frac{\partial u}{\partial x_{2}}\right| \\
& \lesssim h_{2}^{-1}(\operatorname{meas} e)^{1 / q+1-1 / p} \sum_{i=0}^{k}\left(\operatorname{meas} \sigma_{i}\right)^{-1}\left\|\frac{\partial u}{\partial x_{2}} ; L^{p}\left(S_{e}\right)\right\|
\end{aligned}
$$

For pentahedral and hexahedral elements the proof is similar. We only replace (5.4) by

$$
\varphi_{i, j}\left(x_{1}, x_{2}, x_{3}\right)=\phi^{i}\left(x_{1}, x_{2}\right) \phi_{j}\left(x_{3}\right), \quad i=0, \ldots, K, j=0, \ldots, k,
$$

with appropriate basis functions $\phi^{i}\left(x_{1}, x_{2}\right)$ and

$$
K=(k+1)^{2}-1 \quad \text { for hexahedra, } \quad K=\left(\begin{array}{c}
k+2 \\
2
\end{array}\right)-1 \text { for pentahedra }
$$

In the case of simplicial elements we have to modify these considerations slightly. We will explain it in the two-dimensional case. Consider an element $e$ with nodes $X_{i, j}$,

$$
\begin{aligned}
e & =\left\{\left(x_{1}, x_{2}\right): \xi_{1} \leq x_{1} \leq \eta_{1}, \xi_{2} \leq x_{2} \leq \eta_{2}-\left(x_{1}-\xi_{1}\right) \frac{\eta_{2}-\xi_{2}}{\eta_{1}-\xi_{1}}\right\}, \\
X_{i, j} & =\left(\xi_{1}+\frac{i}{k}\left(\eta_{1}-\xi_{1}\right), \xi_{2}+\frac{j}{k}\left(\eta_{2}-\xi_{2}\right)\right),
\end{aligned}
$$




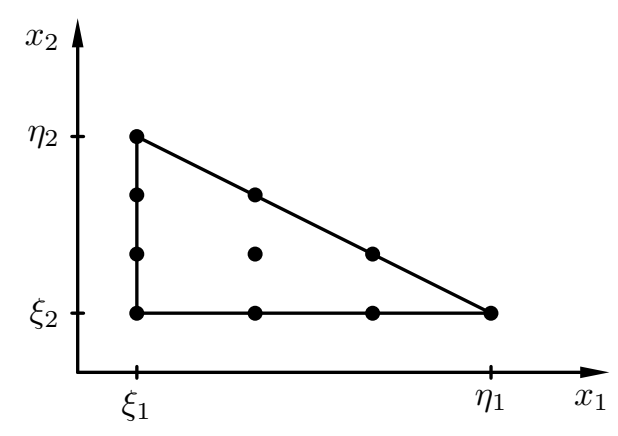

FIGURE 8. Illustration of the case of a triangle.

and nodal basis functions $\varphi_{i, j}, i=0, \ldots, k, j=0, \ldots, k-i$, as illustrated in Figure 8. The new basis functions are

$$
\chi_{i, j}=\sum_{s=0}^{j} \varphi_{i, s}, \quad i=0, \ldots, k, j=0, \ldots, k-i .
$$

We get

$$
\begin{aligned}
\mathrm{L}_{h} u & =\sum_{i=0}^{k} \sum_{j=0}^{k-i} a_{i, j} \varphi_{i, j}=\sum_{i=0}^{k}\left(\sum_{j=0}^{k-i-1}\left(a_{i, j}-a_{i, j+1}\right) \chi_{i, j}+a_{i, k-i} \chi_{i, k-i}\right), \\
\left\|\frac{\partial \mathrm{L}_{h} u}{\partial x_{2}} ; L^{q}(e)\right\| & \lesssim \sum_{i=0}^{k}\left(\sum_{j=0}^{k-i-1}\left|a_{i, j}-a_{i, j+1}\right|\left\|\frac{\partial \chi_{i, j}}{\partial x_{2}} ; L^{q}(e)\right\|+\left|a_{i, k-i}\right|\left\|\frac{\partial \chi_{i, k-i}}{\partial x_{2}} ; L^{q}(e)\right\|\right) .
\end{aligned}
$$

To conclude (5.2) with the same arguments as above it remains to show that

$$
\frac{\partial \chi_{i, k-i}}{\partial x_{2}}=0 \quad \text { for all } i=0, \ldots, k .
$$

For this we observe that $\chi_{i, k-i}$ is uniquely determined by

$$
\chi_{i, k-i}\left(X_{s, j}\right)= \begin{cases}1 & \text { for } s=i, j=0, \ldots, k-i, \\ 0 & \text { else. }\end{cases}
$$

Thus $\chi_{i, k-i}=\phi^{i}\left(x_{1}\right)$ with $\phi^{i}$ in the sense of (5.4), and (5.8) is proved.

The proof for tetrahedral elements is analogous.

Theorem 9. Assume that (5.1) is valid. On anisotropic meshes of tensor-product type the modified Scott-Zhang operator $\mathrm{L}_{h}$ satisfies the following estimates:

$$
\begin{aligned}
\left|\mathrm{L}_{h} u ; W^{m, q}(e)\right| & \lesssim(\text { meas } e)^{1 / q-1 / p}\left|u ; W^{m, p}\left(S_{e}\right)\right| \\
\left|u-\mathrm{L}_{h} u ; W^{m, q}(e)\right| & \lesssim(\operatorname{meas} e)^{1 / q-1 / p} \sum_{|\alpha|=\ell-m} h^{\alpha}\left|D^{\alpha} u ; W^{m, p}\left(S_{e}\right)\right|,
\end{aligned}
$$

$0 \leq m \leq \ell, 1 \leq \ell \leq k+1$, provided that $u \in W^{\ell, p}\left(S_{e}\right)$. For (5.10) the numbers $p, q \in[1, \infty]$ must be such that $W^{\ell, p}(e) \hookrightarrow W^{m, q}(e)$. 
Proof. Estimate (5.10) follows from (5.9) via Lemma 1 as it was done for $\mathrm{S}_{h}$ in the proof of Theorem 6 . So the main point is to prove (5.9). For $m=0$, this can be done as in the proof of (3.9). The case $m=1$ is treated in Lemma 8.

Let $m \geq 2$. Consider a multi-index $\gamma$ with $|\gamma|=m$ and define $m_{2}:=\gamma_{d}, m_{1}:=m-m_{2}$. In the proof of Lemma 8, we made for the case $m_{2}=1$ a transformation of the nodal basis $\varphi_{i, j}$ to a basis $\chi_{i, j}$ in order to obtain differences of first order:

$$
\frac{\partial}{\partial x_{d}} \sum_{i=0}^{K} \sum_{j=0}^{k} a_{i, j} \varphi_{i, j}=\frac{\partial}{\partial x_{d}} \sum_{i=0}^{K} \sum_{j=0}^{k-1}\left(a_{i, j}-a_{i, j+1}\right) \chi_{i, j} .
$$

This process is repeated until differences of order $m_{2}$ are created: For simplicity consider again the onedimensional situation. We define recursively coefficients $a_{i}^{(n)}$ and functions $\chi_{i}^{(n)}, i=0, \ldots, k-n, n=$ $0, \ldots, m_{2}$, by

$$
\begin{array}{ll}
a_{i}^{0}:=a_{i}, \quad a_{i}^{(n+1)}:=a_{i}^{(n)}-a_{i+1}^{(n)}, & i=0, \ldots, k-n, \\
\chi_{i}^{0}:=\varphi_{i}, \quad \chi_{i}^{(n+1)}:=\sum_{s=0}^{i} \chi_{s}^{(n)}, & i=0, \ldots, k,
\end{array}
$$

and obtain

$$
\frac{\partial^{m_{2}}}{\partial x^{m_{2}}} \sum_{i=0}^{k} a_{i} \varphi_{i}=\frac{\partial^{m_{2}}}{\partial x^{m_{2}}} \sum_{i=0}^{k-m_{2}} a_{i}^{\left(m_{2}\right)} \chi_{i}^{\left(m_{2}\right)}
$$

We get this by induction in analogy to the proof of Lemma 8 . The only point is to prove that

$$
\frac{\partial^{n+1}}{\partial x^{n+1}} \chi_{k-n}^{(n+1)}=0 \quad \text { for } n=0, \ldots, m_{2}-1 .
$$

This can be shown for any fixed $n$ via $\chi_{i}^{(n+1)}=\sum_{s=0}^{i}\left(\begin{array}{c}i-s+n \\ n\end{array}\right) \chi_{s}^{(0)}$ (proof by induction) which yields $\chi_{k}^{(n+1)}=$ $\sum_{s=0}^{k}\left(\begin{array}{c}k-s+n \\ n\end{array}\right) \varphi_{s}, \chi_{k}^{(n+1)}\left(X_{r}\right)=\left(\begin{array}{c}k-r+n \\ n\end{array}\right), r=0, \ldots, k, \chi_{k}^{(n+1)} \in \mathcal{P}_{n}^{1}$. From $\chi_{i}^{(n+1)}=\chi_{i+1}^{(n+1)}-\chi_{i+1}^{(n)}$ this gives by induction $\chi_{i}^{(n+1)} \in \mathcal{P}_{n}^{1}$ for $i=k, k-1, \ldots, k-n$. Thus

$$
\frac{\partial^{n+1}}{\partial x^{n+1}} \chi_{i}^{(n+1)}=0 \text { for } i=k-n, \ldots, k \text {. }
$$

Consider now rectangular elements $(d=2)$ and transfer this basis transformation to the $x_{2}$-direction. We derive (again by induction) from (5.11)

$$
\frac{\partial^{m_{2}}}{\partial x_{d}^{m_{2}}} \sum_{i=0}^{k} \sum_{j=0}^{k} a_{i, j} \varphi_{i, j}=\frac{\partial^{m_{2}}}{\partial x_{d}^{m_{2}}} \sum_{i=0}^{k} \sum_{j=0}^{k-m_{2}} a_{i, j}^{\left(m_{2}\right)} \chi_{i, j}^{\left(m_{2}\right)} .
$$

The so created differences $a_{i, j}^{(n+1)}=a_{i, j}^{(n)}-a_{i, j+1}^{(n)}$ are used now to establish an integral representation; compare (5.6):

$$
a_{i, j}^{(1)}=-\int_{\sigma_{i, j}} \psi_{i}\left(x_{1}\right) \int_{0}^{\delta} \frac{\partial u}{\partial x_{d}}\left(x_{1}, y_{j}+\eta_{1}\right) \mathrm{d} \eta_{1} \mathrm{~d} x_{1}
$$


$\delta=y_{j+1}-y_{j}$ is assumed to be independent of $j$. We continue recursively and obtain

$$
\begin{aligned}
a_{i, j}^{(2)} & =-\int_{\sigma_{i, j}} \psi_{i}\left(x_{1}\right)\left[\int_{0}^{\delta} \frac{\partial u}{\partial x_{d}}\left(x_{1}, y_{j}+\eta_{1}\right) \mathrm{d} \eta_{1}-\int_{0}^{\delta} \frac{\partial u}{\partial x_{d}}\left(x_{1}, y_{j+1}+\eta_{1}\right) \mathrm{d} \eta_{1}\right] \mathrm{d} x_{1} \\
& =(-1)^{2} \int_{\sigma_{i, j}} \psi_{i}\left(x_{1}\right) \int_{0}^{\delta} \int_{0}^{\delta} \frac{\partial^{2} u}{\partial x_{d}^{2}}\left(x_{1}, y_{j}+\eta_{1}+\eta_{2}\right) \mathrm{d} \eta_{1} \mathrm{~d} \eta_{2} \mathrm{~d} x_{1}, \\
a_{i, j}^{(n)} & =(-1)^{n} \int_{\sigma_{i, j}} \psi_{i}\left(x_{1}\right) \underbrace{\int_{0}^{\delta} \cdots \int_{0}^{\delta}}_{n \text { times }} \frac{\partial^{n} u}{\partial x_{d}^{n}}\left(x_{1}, y_{j}+\eta_{1}+\cdots+\eta_{n}\right) \mathrm{d} \eta_{1} \cdots \mathrm{d} \eta_{n} \mathrm{~d} x_{1} .
\end{aligned}
$$

Using (3.11) and $\delta \sim h_{2}$ we obtain

$$
\left|a_{i, j}^{(n)}\right| \lesssim\left(\operatorname{meas} \sigma_{i, j}\right)^{-1} h_{d}^{n-1}\left\|\frac{\partial^{n} u}{\partial x_{d}^{n}} ; L^{1}\left(S_{e}\right)\right\|
$$

Replace now meas $\sigma_{i, j}$ by meas $\sigma:=\min _{i, j}$ meas $\sigma_{i, j}$ and $u$ by $u-w, w \in \mathcal{P}_{m-1}^{2}$ arbitrary. Together with (5.12) we conclude that

$$
\begin{aligned}
\left\|D^{\gamma} \mathrm{L}_{h} u ; L^{q}(e)\right\| & =\left\|D^{\gamma} \mathrm{L}_{h}(u-w) ; L^{q}(e)\right\| \\
& \lesssim \sum_{i=0}^{k} \sum_{j=0}^{k-m_{2}}\left|a_{i, j}^{\left(m_{2}\right)}\right|\left\|D^{\gamma} \chi_{i, j}^{\left(m_{2}\right)} ; L^{q}(e)\right\| \\
& \lesssim h^{-\gamma}(\operatorname{meas} e)^{1 / q} \sum_{i=0}^{k} \sum_{j=0}^{k-m_{2}}\left|a_{i, j}^{\left(m_{2}\right)}\right| \\
& \lesssim h^{-\gamma}(\operatorname{meas} e)^{1 / q}(\operatorname{meas} \sigma)^{-1} h_{d}^{m_{2}-1}\left\|\frac{\partial^{m_{2}}}{\partial x_{d}^{m_{2}}}(u-w) ; L^{1}\left(S_{e}\right)\right\| \\
& \lesssim h^{-\gamma} h_{d}^{m_{2}}(\operatorname{meas} e)^{1 / q-1 / p}\left\|\frac{\partial^{m_{2}}}{\partial x_{d}^{m_{2}}}(u-w) ; L^{p}\left(S_{e}\right)\right\| \\
& \lesssim h_{1}^{-m_{1}}(\operatorname{meas} e)^{1 / q-1 / p} \sum_{|\alpha| \leq m-m_{2}} h^{\alpha}\left\|D^{\alpha} \frac{\partial^{m_{2}}}{\partial x_{d}^{m_{2}}}(u-w) ; L^{p}\left(S_{e}\right)\right\| .
\end{aligned}
$$

In order to derive (5.13) we have used that $h_{d}$ meas $\sigma \sim$ meas e. Via Corollary 2, (5.1), and $m=m_{1}+m_{2}$ we obtain

$$
\begin{aligned}
\left\|D^{\gamma} \mathrm{L}_{h} u ; L^{q}(e)\right\| & \lesssim h_{1}^{-m_{1}}(\text { meas } e)^{1 / q-1 / p} \sum_{|\alpha|=m-m_{2}} h^{\alpha}\left\|D^{\alpha} \frac{\partial^{m_{2}} u}{\partial x_{d}^{m_{2}}} ; L^{p}\left(S_{e}\right)\right\| \\
& \leq(\text { meas } e)^{1 / q-1 / p} \sum_{|\alpha|=m-m_{2}}\left\|D^{\alpha} \frac{\partial^{m_{2}} u}{\partial x_{d}^{m_{2}}} ; L^{p}\left(S_{e}\right)\right\| \\
& \leq(\text { meas } e)^{1 / q-1 / p}\left|u ; W^{m, p}\left(S_{e}\right)\right|
\end{aligned}
$$

and (5.9) is proved for rectangular elements. The proof for all other types of elements is similar using the ideas explained in the proof of Lemma 8. 


\section{The operator $\mathrm{E}_{h}$ : Choosing LOng edges in The Three-Dimensional CASe}

\subsection{Stability and approximation in classical Sobolev spaces}

In Sections 4 and 5 we assumed $h_{1} \sim h_{2}$ in the three-dimensional case. We will now investigate the general three-dimensional situation of independent mesh sizes $h_{1}, h_{2}$, and $h_{3}$. But for simplicity, we treat only tensor product meshes in the sense of Definition 2 in Section 2. In order to obtain in Subsection 6.2 a notation which is compatible with that in Subsection 4.2 we let

$$
h_{1} \leq h_{2} \leq h_{3} .
$$

The investigation of the operators $\mathrm{S}_{h}$ and $\mathrm{L}_{h}$ was based on taking $\sigma_{i}$ as isotropic faces, that means that $h_{2}$ is of the same order as $h_{1}$ or $h_{3}$. In [15] it was suggested to overcome this restriction by taking one-dimensional $\sigma_{i}$ but this was not elaborated thoroughly. We will now investigate which estimates can be obtained in this case. We assume the following properties which are analogous to the ones in Section 5.

$\left(\mathrm{P} 1^{\prime}\right) \sigma_{i}$ is parallel to the $x_{3}$-axis.

(P2) $X_{i} \in \overline{\sigma_{i}}$.

$\left(\mathrm{P}^{\prime}\right)$ There exists an edge $\varsigma$ of some element $e$ such that the projection of $\varsigma$ on the $x_{3}$-axis is identical with the projection of $\sigma_{i}$.

$\left(\mathrm{P} 4^{\prime}\right)$ If the projections of any two points $X_{i}$ and $X_{j}$ on the $x_{3}$-axis coincide then so do the projections of $\sigma_{i}$ and $\sigma_{j}$.

The corresponding operator is denoted by $\mathrm{E}_{h}: W^{\ell, p}(\Omega) \rightarrow V_{h}$. Note that it is defined only for $u \in W^{\ell, p}(\Omega)$ with

$$
\ell \geq 2 \text { for } p=1, \quad \ell>\frac{2}{p} \text { otherwise, }
$$

to guarantee that $\left.u\right|_{\sigma_{i}} \in L^{1}\left(\sigma_{i}\right)$. Condition (6.2) can be reformulated to

$$
\ell \geq 2, p \in[1, \infty] \text { or } \ell=1, p \in(2, \infty]
$$

Theorem 10. Consider an element e of a tensor product mesh and assume that (6.1) and (2.4) are fulfilled. Then the operator $\mathrm{E}_{h}$ satisfies for all $q \in[1, \infty]$ the following estimates:

$$
\left|\mathrm{E}_{h} u ; W^{m, q}(e)\right| \lesssim(\text { meas } e)^{1 / q-1 / p} \sum_{|\alpha| \leq 1} h^{\alpha}\left|D^{\alpha} u ; W^{m, p}\left(S_{e}\right)\right|
$$

if $m \geq 1$ or $p>2$, and

$$
\left\|\mathrm{E}_{h} u ; L^{q}(e)\right\| \lesssim(\text { meas } e)^{1 / q-1 / p} \sum_{|\alpha| \leq \ell} h^{\alpha}\left\|D^{\alpha} u ; L^{p}\left(S_{e}\right)\right\|
$$

with $\ell$ and $p$ satisfying (6.3). The approximation error estimate

$$
\left|u-\mathrm{E}_{h} u ; W^{m, q}(e)\right| \lesssim(\text { meas } e)^{1 / q-1 / p} \sum_{|\alpha|=\ell-m} h^{\alpha}\left|D^{\alpha} u ; W^{m, p}\left(S_{e}\right)\right|
$$

holds if $0 \leq m \leq \ell-1 \leq k, p$ satisfies (6.3), $q$ is such that $W^{\ell, p}(e) \hookrightarrow W^{m, q}(e)$, and $u \in W^{\ell, p}\left(S_{e}\right)$.

We will see in the proof that for certain derivatives $D^{\gamma} \mathrm{E}_{h} u$ the stability estimate (6.4) can still be improved. 
Proof. We prove the theorem for brick elements. Other element types are treated similarly, see the discussion in the proof of Lemma 8. We have to consider different cases separately.

First, let $\gamma$ be a multi-index with $|\gamma|=m$ and $\gamma_{1} \neq 0, \gamma_{2} \neq 0$. We use the difference technique developed in the proof of Theorem 9 for both directions $x_{1}$ and $x_{2}$. In analogy to (5.13) we obtain for all $w \in \mathcal{P}_{m-1}^{3}$

$$
\begin{aligned}
\left\|D^{\gamma} \mathrm{E}_{h} u, L^{q}(e)\right\| & =\left\|D^{\gamma} \mathrm{E}_{h}(u-w), L^{q}(e)\right\| \\
& \lesssim h^{-\gamma} h_{1}^{\gamma_{1}} h_{2}^{\gamma_{2}}(\operatorname{meas} e)^{1 / q-1 / p}\left\|\frac{\partial^{\gamma_{1}}}{\partial x_{1}^{\gamma_{1}}} \frac{\partial^{\gamma_{2}}}{\partial x_{2}^{\gamma_{2}}}(u-w) ; L^{p}\left(S_{e}\right)\right\| \\
& \leq h_{3}^{-\gamma_{3}}(\operatorname{meas} e)^{1 / q-1 / p} \sum_{|\alpha| \leq \gamma_{3}} h^{\alpha}\left|D^{\alpha} u ; W^{\gamma_{1}+\gamma_{2}, p}\left(S_{e}\right)\right| .
\end{aligned}
$$

Using Corollary 2 and (6.1) we conclude

$$
\begin{aligned}
\left\|D^{\gamma} \mathrm{E}_{h} u, L^{q}(e)\right\| & \lesssim h_{3}^{-\gamma_{3}}(\operatorname{meas} e)^{1 / q-1 / p} \sum_{|\alpha|=\gamma_{3}} h^{\alpha}\left|D^{\alpha} u ; W^{\gamma_{1}+\gamma_{2}, p}\left(S_{e}\right)\right| \\
& \leq(\text { meas } e)^{1 / q-1 / p}\left|u ; W^{m, p}\left(S_{e}\right)\right| .
\end{aligned}
$$

In a second case we assume $\gamma_{n} \neq 0, n=1$ or $n=2$, but $\gamma_{3-n}=0, \gamma_{3} \neq 0$. Then we can use the difference technique only within some faces $f_{i}(i=0, \ldots, k)$ which are parallel to the $x_{n}, x_{3}$-plane. Defining $f:=\bigcup_{i=0}^{k} f_{i}$ we find as above that for all $w \in \mathcal{P}_{m-1}^{3}$

$$
\begin{aligned}
\left\|D^{\gamma} \mathrm{E}_{h} u, L^{q}(e)\right\| & =\left\|D^{\gamma} \mathrm{E}_{h}(u-w), L^{q}(e)\right\| \\
& \lesssim h^{-\gamma} h_{n}^{\gamma_{n}}(\text { meas } e)^{1 / q}(\operatorname{meas} f)^{-1 / p}\left\|\frac{\partial^{\gamma_{n}}}{\partial x_{n}^{\gamma_{n}}}(u-w) ; L^{p}(f)\right\|
\end{aligned}
$$

Using the trace theorem $W^{\gamma_{3}, p}\left(S_{e}\right) \hookrightarrow L^{p}(f)$ and again Corollary 2 as well as (6.1) we obtain

$$
\begin{aligned}
\left\|D^{\gamma} \mathrm{E}_{h} u, L^{q}(e)\right\| & \lesssim h_{3}^{-\gamma_{3}}(\text { meas } e)^{1 / q-1 / p} \sum_{|\alpha| \leq \gamma_{3}} h^{\alpha}\left|D^{\alpha}(u-w) ; W^{\gamma_{n}, p}\left(S_{e}\right)\right| \\
& \lesssim h_{3}^{-\gamma_{3}}(\operatorname{meas} e)^{1 / q-1 / p} \sum_{|\alpha|=\gamma_{3}} h^{\alpha}\left|D^{\alpha} u ; W^{\gamma_{n}, p}\left(S_{e}\right)\right| \\
& \leq(\text { meas } e)^{1 / q-1 / p}\left|u ; W^{m, p}\left(S_{e}\right)\right| .
\end{aligned}
$$

Consider now the remaining pure derivatives. Let first be $\gamma_{n}=m, n=1$ or $n=2, \gamma_{3}=0$. Estimate (6.7) holds in this case as well. By using $p=1$ and $w=0$ it reads now

$$
\left\|D^{\gamma} \mathrm{E}_{h} u, L^{q}(e)\right\| \lesssim(\text { meas } e)^{1 / q}(\text { meas } f)^{-1}\left\|D^{\gamma} u ; L^{1}(f)\right\|
$$

With the trace theorem $W^{1, p}\left(S_{e}\right) \hookrightarrow L^{1}(f)$ for all $p \in[1, \infty]$ we conclude the assertion (6.4).

Finally, for $\gamma_{3}=m, \gamma_{1}=\gamma_{2}=0$, the proof of the stability is completely analogous to the proof of Lemma 4 . We have for all $w \in \mathcal{P}_{m-1}^{3}$

$$
\left\|D^{\gamma} \mathrm{E}_{h} u, L^{q}(e)\right\| \lesssim h_{3}^{-m}(\text { meas } e)^{1 / q} \sum_{i \in I_{e}}\left(\operatorname{meas} \sigma_{i}\right)^{-1}\left\|u-w ; L^{1}\left(\sigma_{i}\right)\right\|
$$


The trace theorem $W^{m+1, p}\left(S_{e}\right) \hookrightarrow L^{1}\left(\sigma_{i}\right)$ (which is the reason for the assumption $m \geq 1$ or $p>2$ ) and Corollary 2 yield

$$
\begin{aligned}
\left\|D^{\gamma} \mathrm{E}_{h} u, L^{q}(e)\right\| & \lesssim h_{3}^{-m}(\text { meas } e)^{1 / q-1 / p} \sum_{|\alpha| \leq m} \sum_{|\beta| \leq 1} h^{\alpha+\beta}\left\|D^{\alpha+\beta}(u-w) ; L^{p}\left(S_{e}\right)\right\| \\
& \lesssim h_{3}^{-m}(\text { meas } e)^{1 / q-1 / p} \sum_{|\alpha|=m|\beta| \leq 1} \sum_{\mid \beta} h^{\alpha+\beta}\left\|D^{\alpha+\beta} u ; L^{p}\left(S_{e}\right)\right\| \\
& \lesssim(\text { meas } e)^{1 / q-1 / p} \sum_{|\beta| \leq 1} h^{\beta}\left|D^{\beta} u ; W^{m, p}\left(S_{e}\right)\right| .
\end{aligned}
$$

Note that in this last case $\left(\gamma_{3}=m\right)$ for $m \geq 2$ and for $m=1, p>2$, it can even be proved that

$$
\left\|D^{\gamma} \mathrm{E}_{h} u, L^{q}(e)\right\| \lesssim(\text { meas } e)^{1 / q-1 / p}\left|u ; W^{m, p}\left(S_{e}\right)\right|
$$

because then $W^{m, p}\left(S_{e}\right) \hookrightarrow L^{1}\left(\sigma_{i}\right)$ holds.

Estimate (6.5) is trivial since

$$
\left\|\mathrm{E}_{h} u, L^{q}(e)\right\| \lesssim(\operatorname{meas} e)^{1 / q} \sum_{i \in I_{e}}\left(\operatorname{meas} \sigma_{i}\right)^{-1}\left\|u ; L^{1}\left(\sigma_{i}\right)\right\|,
$$

and the embedding $W^{\ell, p}\left(S_{e}\right) \hookrightarrow L^{1}\left(\sigma_{i}\right)$ holds just for $\ell, p$ satisfying (6.3).

Estimate (6.6) is concluded from $(6.4,6.5)$ as in the proof of Theorem 6.

It is interesting to point out that the proof shows that

$$
\left\|D^{\gamma} \mathrm{E}_{h} u, L^{q}(e)\right\| \lesssim(\operatorname{meas} e)^{1 / q-1 / p}\left|u ; W^{m, p}\left(S_{e}\right)\right|
$$

holds for $\gamma$ with $|\gamma|=m$ if at most one of the numbers $\gamma_{1}, \gamma_{2}, \gamma_{3}$ vanishes. Our way of proof does not work for pure derivatives. Consider for example the case $\gamma=(1,0,0)$. To prove (6.9) with $p>2\left(\mathrm{E}_{h} u\right.$ is defined only for $u \in W^{1, p}(\Omega)$ with $p>2$.) one would have to skip the trace on $f$ and to use a trace theorem in the form (3.13). But this leads to

$$
\left\|D^{\gamma} \mathrm{E}_{h} u, L^{q}(e)\right\| \lesssim h_{1}^{-1}(\text { meas } e)^{1 / q-1 / p} \sum_{|\alpha| \leq 1} h^{\alpha}\left\|D^{\alpha} u ; L^{p}\left(S_{e}\right)\right\|
$$

with some diverging terms at the right-hand side. The case $\gamma=(1,0,0)$ would be tractable only if

$$
\left\|D^{\gamma} \mathrm{E}_{h} u, L^{q}(e)\right\| \lesssim(\text { meas } e)^{1 / q-1 / p}\left\|D^{\gamma} u ; L^{p}\left(S_{e}\right)\right\|
$$

was valid. It is not clear whether this estimate holds.

Remark 1. Our motivation for introducing the operator $\mathrm{E}_{h}$ was to be able to treat the general case of three independent mesh sizes $h_{1} \leq h_{2} \leq h_{3}$. Of course this includes the special case $h_{1} \sim h_{2}$. We point out that in this case the transformation (2.4) can be generalized to $(2.2,2.3)$. To see that then the statement of Theorem 10 is still true consider an arbitrary element $e \in \mathcal{T}_{h}$ and denote its projection into the $x_{1}, x_{2}$-plane by $\zeta$. Because $\mathcal{T}_{h}$ is of tensor product type, and because all $\sigma_{i}$ are perpendicular to the $x_{1}, x_{2}$-plane, it suffices to choose $S_{e}$ such that its projection to the $x_{1}, x_{2}$-plane is again $\zeta$ (and $\sigma_{i} \subset \overline{S_{e}}$ ), compare Figure 9. Via the transformation

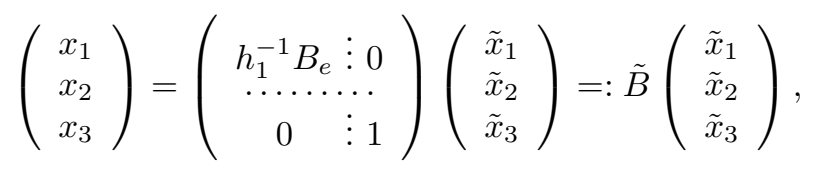



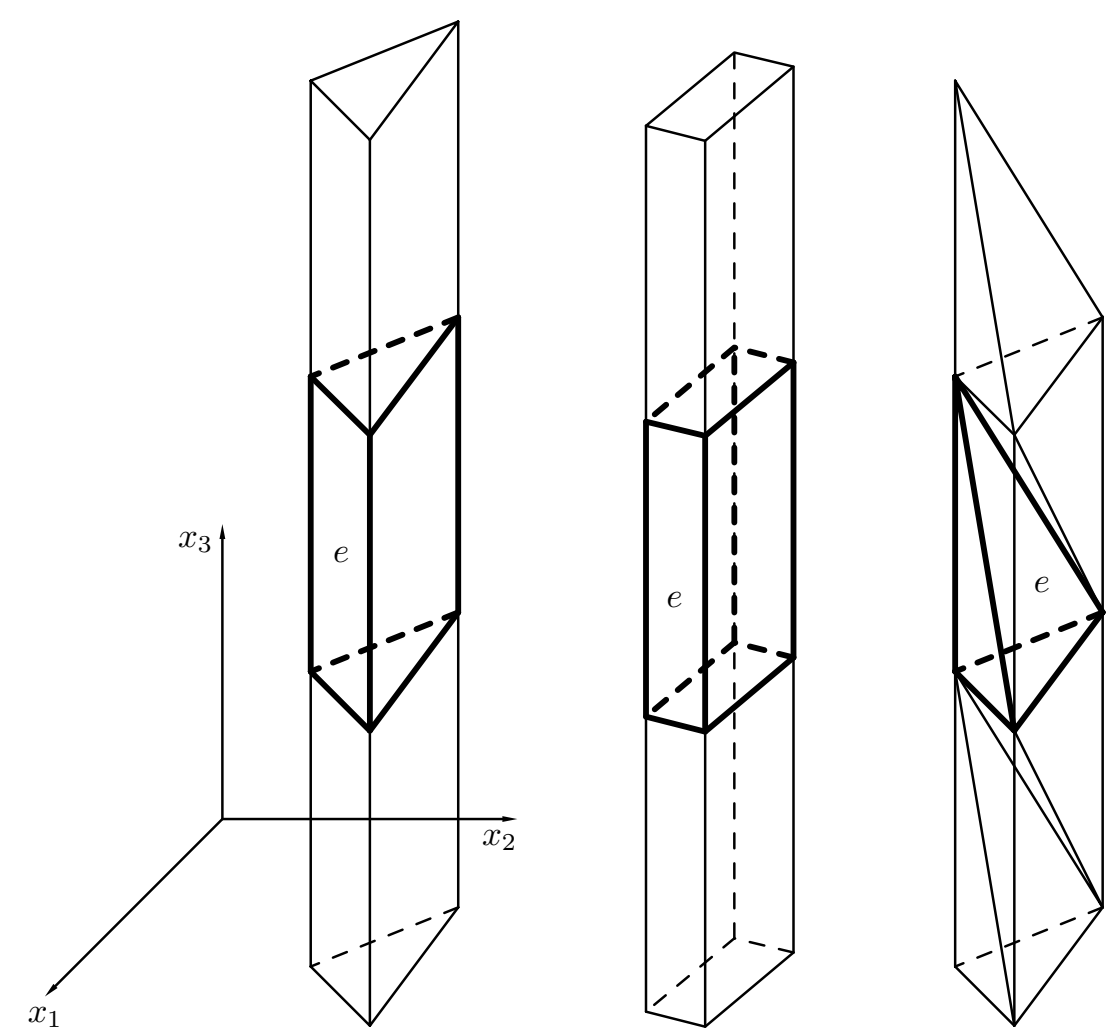

Figure 9. Illustration of the possible choice of a smaller $S_{e}$ in the case of $\mathrm{E}_{h}$ (three element types).

$B_{e}$ from (2.2), the domains $e$ and $S_{e}$ can be mapped to $\tilde{e}$ and $\tilde{S}_{e}=S_{\tilde{e}}$ which satisfy (locally) the assumptions made at the beginning of this section. That means that Theorem 10 holds true with respect to the coordinate system $\tilde{x}_{1}, \tilde{x}_{2}, \tilde{x}_{3}$. By observing that

$$
\operatorname{det} \tilde{B} \sim 1, \quad\|\tilde{B}\| \sim 1, \quad\left\|\tilde{B}^{-1}\right\| \sim 1
$$

we find that Theorem 10 extends to the meshes described above.

\subsection{Stability in weighted Sobolev spaces}

As in Subsection 4.2 we do not have an estimate of type $(1,1)$ for $\mathrm{E}_{h}$. Therefore we consider a stability estimate for functions from weighted Sobolev spaces $V_{\beta}^{\ell, p}\left(S_{e}\right)$. These spaces were introduced in $(4.9,4.10)$. To be able to apply the transformation (2.4) to the weight we will restrict the consideration to the case $h_{1} \sim h_{2}$. However, we can then relax (2.4) to (2.2), see Remark 1.

Lemma 11. Consider an element e of a tensor product mesh and assume that (6.1) and (2.4) are fulfilled. Let $m$ be an integer and $\beta, p, q$ be real numbers with $0 \leq m \leq k, p, q \in[1, \infty], \beta<2-2 / p, \beta \leq 1$. Then for $u \in W^{m, p}\left(S_{e}\right) \cap V_{\beta}^{m+1, p}\left(S_{e}\right)$ the stability estimate

$$
\left|\mathrm{E}_{h} u ; W^{m, q}(e)\right| \leq(\operatorname{meas} e)^{1 / q-1 / p} h_{1}^{-\beta} \sum_{|\alpha|=m-1} \sum_{|t|=1} h^{t}\left\|D^{\alpha+t} v ; V_{\beta}^{1, p}\left(S_{e}\right)\right\|
$$

holds if $m \geq 1$ or $p \geq 2$. 
Proof. Observe that the relations

$$
\begin{aligned}
\left\|v ; L^{1}\left(S_{e}\right)\right\| & \leq\left\|r^{-\beta} ; L^{p^{\prime}}\left(S_{e}\right)\right\|\left\|r^{\beta} v ; L^{p}\left(S_{e}\right)\right\|, \\
\left\|r^{-\beta} ; L^{p^{\prime}}\left(S_{e}\right)\right\| & \lesssim\left(\operatorname{meas} S_{e}\right)^{1-1 / p} h_{1}^{-\beta}
\end{aligned}
$$

(compare $(4.15,4.16))$ lead to the embedding

$$
V_{\beta}^{m+1, p}\left(S_{e}\right) \hookrightarrow V_{0}^{m+1,1}\left(S_{e}\right) \hookrightarrow W^{m+1,1}\left(S_{e}\right), \quad \beta<2-\frac{2}{p},
$$

that means $u \in W^{m+1,1}\left(S_{e}\right)$. Therefore we can apply Theorem 10 (see also Rem. 1) with $p=1$ :

$$
\left|\mathrm{E}_{h} u ; W^{m, q}(e)\right| \lesssim(\operatorname{meas} e)^{1 / q-1} \sum_{|\alpha| \leq 1} h^{\alpha}\left|D^{\alpha} u ; W^{m, 1}\left(S_{e}\right)\right| .
$$

Notice further that $(6.11,6.12)$ lead to the estimate

$$
\left\|v ; L^{1}\left(S_{e}\right)\right\| \lesssim\left(\operatorname{meas} S_{e}\right)^{1-1 / p} h_{1}^{-\beta}\left\|r^{\beta} v ; L^{p}\left(S_{e}\right)\right\|, \quad \beta<2-\frac{2}{p} .
$$

So we get

$$
\begin{aligned}
& \sum_{|\alpha| \leq 1} \sum_{|t|=1} h^{\alpha}\left\|D^{\alpha+t} v ; L^{1}\left(S_{e}\right)\right\| \\
& \lesssim\left(\operatorname{meas} S_{e}\right)^{1-1 / p} h_{1}^{-\beta}\left(\sum_{|\alpha|=1} \sum_{|t|=1} h^{\alpha}\left\|r^{\beta} D^{\alpha+t} v ; L^{p}\left(S_{e}\right)\right\|+\sum_{|t|=1} h_{1}\left\|r^{\beta-1} D^{t} v ; L^{p}\left(S_{e}\right)\right\|\right) \\
& \lesssim\left(\operatorname{meas} S_{e}\right)^{1-1 / p} h_{1}^{-\beta} \sum_{|s|=1} h^{s}\left\|D^{s} v ; V^{1, p}\left(S_{e}\right)\right\| .
\end{aligned}
$$

Together with (6.13) the assertion (6.10) is concluded.

\section{Application to the Poisson problem in a domain with an edge}

Consider the Poisson problem with in general mixed boundary conditions in a three-dimensional polyhedral domain $\Omega$. It is well known that the solution has in general singularities near corners and edges and near the lines where the type of the boundary condition changes. As a result, the finite element method on quasiuniform meshes loses accuracy. The rate of convergence is smaller in comparison with that for problems with smooth solutions. To compensate this, specially adapted numerical methods have been developed. The singular function method which is well developed for two-dimensional problems, is used for three-dimensional problems in $[14,26]$. However, mesh refinement techniques seem to be easier to handle. Refined isotropic meshes were considered in $[5,12,25]$ for the finite element method and the boundary element method but this approach leads to overrefinement near edges. This overrefinement can be avoided by using anisotropic meshes in the neighbourhood of the edges $[4,9,29]$.

In $[4,9]$ we considered the Dirichlet problem for the Poisson equation over a prismatic domain

$$
\Omega=G \times Z
$$

where $G \subset \mathbb{R}^{2}$ is a bounded polygonal domain and $Z:=\left(0, z_{0}\right) \subset \mathbb{R}$ is an interval. This restriction was made there because we wanted to focus on edge singularities, and such domains do not introduce additional corner 
singularities $[33,34]$. The finite element meshes in $[4,9]$ were of tensor product type, graded perpendicularly to the edge and quasi-uniform in the edge direction. Pentahedral meshes seem to be natural but in that papers the pentahedra were divided into three tetrahedra each. Pentahedral elements were used in [10], an unpublished version of the paper [9]. Note that this class of domains and the meshes exactly match the assumptions made in Section 2 for the present paper.

The estimation of the finite element error in the energy norm can be reduced to a general approximation problem due to the projection property of the finite element method. In the previous papers the interpolation error $u-\mathrm{I}_{h} u$ was investigated and it was shown that the family of meshes considered there is suited for the treatment of edge singularities. However, two points are still insufficient: First, the assumptions on the regularity of the right-hand side $f$ of the Poisson equation were quite high in [4]. This drawback was partially removed in [9], but the case $f \in L^{2}(\Omega)$ is still not treated. This is deficient because Nitsche's method for obtaining an $L^{2}(\Omega)$-estimate of the finite element error is not applicable. Second, the refinement condition in [9] is slightly stronger than in [4]; this seems to be unnecessary. The aim of this section is to prove optimal estimates of the finite element error in the $W^{1,2}(\Omega)$ - and the $L^{2}(\Omega)$-norm for $f \in L^{2}(\Omega)$ and the weaker refinement condition of [4]. This is now possible due to the local anisotropic estimates for the quasi-interpolation operators $\mathrm{S}_{h}$ and $\mathrm{E}_{h}$. We point out that one essential ingredient of the proof of these optimal global error estimate is the anisotropic local estimate

$$
\left|u-\mathrm{Q}_{h} u ; W^{1,2}(e)\right| \lesssim \sum_{|\alpha|=1} h_{e}^{\alpha}\left|D^{\alpha} u ; W^{1,2}\left(S_{e}\right)\right| .
$$

This estimate is neither satisfied for $\mathrm{Q}_{h}=\mathrm{I}_{h}$ (see [4]) nor for $\mathrm{Q}_{h}=\mathrm{Z}_{h}, \mathrm{Q}_{h}=\mathrm{C}_{h}$, or $\mathrm{Q}_{h}=\mathrm{O}_{h}$, see the discussion in Section 3.

The plan of this section is the following. First we pose two model problems which differ in their boundary conditions. Then we introduce the family of finite element meshes. The global quasi-interpolation error is estimated in the $W^{1,2}(\Omega)$-seminorm. Because in general the operators do not preserve Dirichlet boundary conditions the model problems are chosen such that in one case $\mathrm{S}_{h}$ and in the other case $\mathrm{E}_{h}$ are appropriate and no modification of the operator is necessary near the boundary. The main result of this section can then be concluded, namely the finite element error estimates. Some remarks on other than the model problems complete this section.

Consider a prismatic domain $\Omega$ as described in (7.1) and denote $\Gamma_{\mathrm{B}}:=\left\{x \in \partial \Omega: x_{3}=0\right.$ or $\left.x_{3}=z_{0}\right\}$ and $\Gamma_{\mathrm{M}}:=\left\{x \in \partial \Omega: 0<x_{3}<z_{0}\right\}=\partial \Omega \backslash \Gamma_{\mathrm{B}}$. Then we treat the mixed boundary value problems

$$
\begin{aligned}
& -\Delta u=f \text { in } \Omega, \quad u=0 \text { on } \Gamma_{\mathrm{B}}, \quad \frac{\partial u}{\partial n}=0 \text { on } \Gamma_{\mathrm{M}}, \\
& -\Delta u=f \text { in } \Omega, \quad u=0 \text { on } \Gamma_{\mathrm{M}}, \quad \frac{\partial u}{\partial n}=0 \text { on } \Gamma_{\mathrm{B}},
\end{aligned}
$$

with $f \in L^{2}(\Omega)$. We assume that the cross-section $G$ has only one corner with interior angle $\omega>\pi$ at the origin; thus $\Omega$ has only one "singular edge" which is part of the $x_{3}$-axis. The case of more than one singular edge introduces no additional difficulties because the edge singularities are of local nature.

Let $V_{0} \subset W^{1,2}(\Omega)$ be the space of all $W^{1,2}(\Omega)$-functions which vanish at the Dirichlet part of the boundary (different for problems $(7.2,7.3)$ ), and introduce the bilinear form $a(.,):. V_{0} \times V_{0} \rightarrow \mathbb{R}$ and the linear form $(f,):. V_{0} \rightarrow \mathbb{R}$ by

$$
a(u, v):=\int_{\Omega} \nabla u \cdot \nabla v, \quad(f, v):=\int_{\Omega} f v .
$$

The variational form of problems $(7.2,7.3)$ is given by

$$
\text { Find } u \in V_{0} \text { such that } a(u, v)=(f, v) \text { for all } v \in V_{0} \text {. }
$$




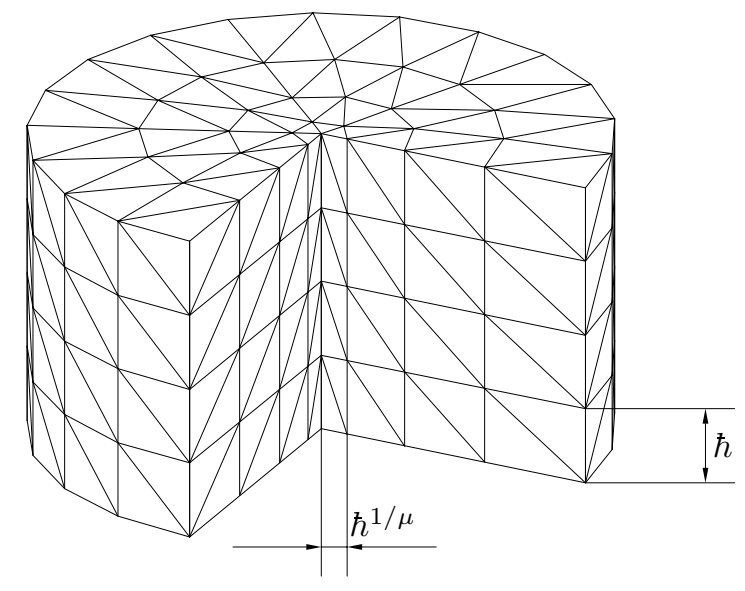

Figure 10. Example for an anisotropic mesh.

The existence of a unique variational solution $u$ follows from the Lax-Milgram lemma.

The properties of the solution $u$ can be described favourably using the weighted Sobolev spaces $V_{\beta}^{\ell, p}$ introduced in Subsection 4.2 .

Lemma 12. The solutions $u$ of both problems (7.2) and (7.3) satisfy

$$
\begin{aligned}
\frac{\partial u}{\partial x_{i}} \in V_{\beta}^{1,2}(\Omega), & \left\|\frac{\partial u}{\partial x_{i}} ; V_{\beta}^{1,2}(\Omega)\right\| & \lesssim\left\|f ; L^{2}(\Omega)\right\|, \quad i=1,2, \beta>1-\frac{\pi}{\omega}, \\
\frac{\partial u}{\partial x_{3}} \in V_{0}^{1,2}(\Omega), & \left\|\frac{\partial u}{\partial x_{3}} ; V_{0}^{1,2}(\Omega)\right\| & \lesssim\left\|f ; L^{2}(\Omega)\right\| .
\end{aligned}
$$

Proof. The singularity of the edge at the $x_{3}$-axis can be described by $(7.5,7.6)$, see for example $\S 26$ and $\S 30$ in [23] or Section 2 in [9]. One can show by mirror techniques that the corners (see also [33,34] for a different proof) and edges at the bottom and the top face do not introduce singularities. Finally, the remaining edges parallel to $x_{3}$-axis were assumed to have an opening angle smaller than $\pi$ such that no singularity occurs.

We define now a family of meshes $\mathcal{T}_{h}=\{e\}$ of tensor product type by introducing in $G$ the standard mesh grading for two-dimensional corner problems, see for example [27]. Let $\{\eta\}$ be a regular isotropic triangulation of $G$; the elements are triangles. With $\hbar$ being the global mesh parameter, $\mu \in(0,1]$ being the grading parameter, $r_{\eta}$ being the distance of $\eta$ to the corner,

$$
r_{\eta}:=\min _{\left(x_{1}, x_{2}\right) \in \bar{\eta}}\left(x_{1}^{2}+x_{2}^{2}\right)^{1 / 2},
$$

and some constant $R>0$, we assume that the element size $h_{\eta}:=\operatorname{diam} \eta$ satisfies

$$
h_{\eta} \sim \begin{cases}\hbar^{1 / \mu} & \text { for } r_{\eta}=0 \\ \hbar r_{\eta}^{1-\mu} & \text { for } 0<r_{\eta} \leq R \\ \hbar & \text { for } r_{\eta}>R\end{cases}
$$

This graded two-dimensional mesh is now extended in the third dimension using a uniform mesh size $\hbar$. In this way we obtain a pentahedral or, by dividing each pentahedron, a tetrahedral triangulation of $\Omega$, see Figure 10 for an illustration. Note that the number of elements is of the order $\hbar^{-3}$ for the full range of $\mu$. The notation 
is extended to the three-dimensional case as follows. Let $r_{e}$ be the distance of an element $e$ to the edge $\left(x_{3}\right.$-axis). Then the element sizes satisfy

$$
h_{1, e} \sim h_{2, e} \sim \begin{cases}\hbar^{1 / \mu} & \text { for } r_{e}=0 \\ \hbar r_{e}^{1-\mu} & \text { for } 0<r_{e} \leq R, \quad h_{3, e} \sim \hbar \\ \hbar & \text { for } r_{e}>R .\end{cases}
$$

We introduce now the finite element space $V_{0 h}:=V_{h} \cap V_{0}$ where $V_{h}$ is defined in Section 1 . The finite element solution $u_{h}$ is determined by

$$
\text { Find } u_{h} \in V_{0 h} \text { such that } a\left(u_{h}, v_{h}\right)=\left(f, v_{h}\right) \text { for all } v_{h} \in V_{0 h} \text {. }
$$

Remember that $V_{0 h}$ is adapted to the Dirichlet boundary condition and therefore different for problems $(7.2,7.3)$.

Theorem 13. Let $u$ be the solution of (7.2). Then the estimate

$$
\left|u-\mathrm{S}_{h} u ; W^{1,2}(\Omega)\right| \lesssim \hbar\left\|f ; L^{2}(\Omega)\right\|
$$

holds if $\mu<\pi / \omega$.

Proof. We reduce the estimation of the global error to the evaluation of the local errors and distinguish between the elements far from the edge $M$ and the elements close to $M$.

For all elements $e$ with $\overline{S_{e}} \cap M=\emptyset$ we can use Theorem 6 with $m=k=1$ and $\ell=p=q=2$ :

$$
\begin{aligned}
\left|u-\mathrm{S}_{h} u ; W^{1,2}(e)\right| & \lesssim \sum_{|\alpha|=1} h_{e}^{\alpha}\left|D^{\alpha} u ; W^{1,2}\left(S_{e}\right)\right| \\
& \lesssim \sum_{i=1}^{2} h_{i, e} r_{e}^{-\beta}\left|\frac{\partial u}{\partial x_{i}} ; V_{\beta}^{1,2}\left(S_{e}\right)\right|+h_{3, e}\left|\frac{\partial u}{\partial x_{3}} ; V_{0}^{1,2}\left(S_{e}\right)\right|
\end{aligned}
$$

for any $\beta>1-\pi / \omega$. Here, we have used the fact that $r_{e} \lesssim \operatorname{dist}\left(S_{e}, M\right)$ holds, which follows from

$$
r_{e} \leq \operatorname{dist}\left(S_{e}, M\right)+h_{1, e^{\prime}} \sim \operatorname{dist}\left(S_{e}, M\right)+\hbar\left[\operatorname{dist}\left(S_{e}, M\right)\right]^{1-\mu}
$$

for sufficiently small $\hbar$. We apply now the assumption (7.7) and obtain for $r_{e} \leq R$ and $\beta=1-\mu$ the relation $h_{i, e} r_{e}^{-\beta} \sim \hbar r_{e}^{1-\mu-\beta}=\hbar(i=1,2)$. The choice $\beta=1-\mu$ is admissible due to the refinement condition $\mu<\pi / \omega$. - In the case $r_{e}>R$ we have $h_{i, e} r_{e}^{-\beta} \lesssim \hbar R^{-\beta} \sim \hbar$. Combining this with (7.9) we obtain

$$
\left|u-\mathrm{S}_{h} u ; W^{1,2}(e)\right| \lesssim \hbar \sum_{i=1}^{2}\left|\frac{\partial u}{\partial x_{i}} ; V_{\beta}^{1,2}\left(S_{e}\right)\right|+\hbar\left|\frac{\partial u}{\partial x_{3}} ; V_{0}^{1,2}\left(S_{e}\right)\right|
$$

Consider now the elements $e$ with $\overline{S_{e}} \cap M \neq \emptyset$. We use the triangle inequality and Lemma 7 with $m=k=1$, $p=q=2, \beta \in(1-\pi / \omega, 1)$ :

$$
\begin{aligned}
\left|u-\mathrm{S}_{h} u ; W^{1,2}(e)\right| & \lesssim\left|u ; W^{1,2}(e)\right|+\left|\mathrm{S}_{h} u ; W^{1,2}(e)\right| \\
& \lesssim \sum_{|\alpha|=1}\left\|D^{\alpha} u, L^{2}(e)\right\|+h_{1, e}^{-\beta} \sum_{|\alpha|=1} h_{e}^{\alpha}\left\|D^{\alpha} u, V_{\beta}^{1,2}\left(S_{e}\right)\right\| .
\end{aligned}
$$


For the first term we use that $r \lesssim h_{1, e}$ in $e$ and $1-\beta>0$ and obtain

$$
\begin{aligned}
\sum_{|\alpha|=1}\left\|D^{\alpha} u, L^{2}(e)\right\| & \lesssim \sum_{i=1}^{2} h_{1, e}^{1-\beta}\left\|\frac{\partial u}{\partial x_{i}} ; V_{\beta-1}^{0,2}(e)\right\|+h_{1, e}\left\|\frac{\partial u}{\partial x_{3}} ; V_{-1}^{0,2}(e)\right\| \\
& \lesssim \hbar \sum_{i=1}^{2}\left\|\frac{\partial u}{\partial x_{i}} ; V_{\beta}^{1,2}(e)\right\|+\hbar\left\|\frac{\partial u}{\partial x_{3}} ; V_{0}^{1,2}(e)\right\| .
\end{aligned}
$$

We also used that $h_{1, e}^{1-\beta} \sim \hbar^{(1-\beta) / \mu}=\hbar$ for $\beta=1-\mu$. The second term is treated with similar arguments:

$$
\begin{aligned}
h_{1, e}^{-\beta} \sum_{|\alpha|=1} h_{e}^{\alpha}\left\|D^{\alpha} u, V_{\beta}^{1,2}\left(S_{e}\right)\right\| & \lesssim \sum_{i=1}^{2} h_{1, e}^{1-\beta}\left\|\frac{\partial u}{\partial x_{i}} ; V_{\beta}^{1,2}\left(S_{e}\right)\right\|+h_{1, e}^{-\beta} \hbar\left\|\frac{\partial u}{\partial x_{3}} ; V_{\beta}^{1,2}\left(S_{e}\right)\right\| \\
& \lesssim \hbar \sum_{i=1}^{2}\left\|\frac{\partial u}{\partial x_{i}} ; V_{\beta}^{1,2}\left(S_{e}\right)\right\|+\hbar\left\|\frac{\partial u}{\partial x_{3}} ; V_{0}^{1,2}\left(S_{e}\right)\right\|
\end{aligned}
$$

The last term was estimated using $r^{\beta} \leq h_{1, e}^{\beta}$.

Inserting $(7.12,7.13)$ in $(7.11)$ we find that $(7.10)$ (with full norms instead of seminorms at the right-hand side) holds for elements with $\overline{S_{e}} \cap M \neq \emptyset$ as well. Summing up over all elements we obtain

$$
\left|u-\mathrm{S}_{h} u ; W^{1,2}(\Omega)\right| \lesssim \hbar \sum_{i=1}^{2}\left\|\frac{\partial u}{\partial x_{i}} ; V_{\beta}^{1,2}(\Omega)\right\|+\hbar\left\|\frac{\partial u}{\partial x_{3}} ; V_{0}^{1,2}(\Omega)\right\|,
$$

$\beta=1-\mu \in(1-\pi / \omega, 1)$. Here we used that only a finite number (independent of $\hbar$ ) of patches $S_{e}$ overlap. By applying Lemma 12 the theorem is proved.

Theorem 14. Let $u$ be the solution of (7.3). Then the estimate

$$
\left|u-\mathrm{E}_{h} u ; W^{1,2}(\Omega)\right| \lesssim \hbar\left\|f ; L^{2}(\Omega)\right\|
$$

holds if $\mu<\pi / \omega$.

Proof. The theorem can be proved in the same way as Theorem 7.2. Note that we used only the following properties of $\mathrm{S}_{h}$ :

$$
\begin{aligned}
\left|u-\mathrm{S}_{h} u ; W^{1,2}(e)\right| & \lesssim \sum_{|\alpha|=1} h_{e}^{\alpha}\left|D^{\alpha} u ; W^{1,2}\left(S_{e}\right)\right|, \\
\left|\mathrm{S}_{h} u ; W^{1,2}(e)\right| & \lesssim h_{1, e}^{-\beta} \sum_{|\alpha|=1}\left\|D^{\alpha} u, V_{\beta}^{1,2}\left(S_{e}\right)\right\| .
\end{aligned}
$$

Both estimates hold true for $\mathrm{E}_{h}$ as well, see Theorem 10 and Lemma 11.

Corollary 15. Let $u$ be the solution of (7.2) or (7.3) and let $u_{h}$ be the finite element solution defined by (7.8). Assume that the mesh is refined according to $\mu<\pi / \omega$. Then the finite element error can be estimated by

$$
\begin{aligned}
\left|u-u_{h} ; W^{1,2}(\Omega)\right| & \lesssim \hbar\left\|f ; L^{2}(\Omega)\right\|, \\
\left\|u-u_{h} ; L^{2}(\Omega)\right\| & \lesssim \hbar^{2}\left\|f ; L^{2}(\Omega)\right\| .
\end{aligned}
$$


Proof. The first estimate follows from Theorems 13 and 14 via the projection property of the finite element method. Note that $\mathrm{S}_{h} u \in V_{0 h}$ in the case of problem (7.2) and $\mathrm{E}_{h} u \in V_{0 h}$ for (7.3). The $L^{2}(\Omega)$-estimate is obtained by Nitsche's method.

By analogy one can prove for $\pi / \omega<\mu \leq 1$ that

$$
\begin{aligned}
\left|u-u_{h} ; W^{1,2}(\Omega)\right| & \lesssim \hbar^{\pi /(\mu \omega)-\varepsilon}\left\|f ; L^{2}(\Omega)\right\|, \\
\left\|u-u_{h} ; L^{2}(\Omega)\right\| & \lesssim \hbar^{2[\pi /(\mu \omega)-\varepsilon]}\left\|f ; L^{2}(\Omega)\right\|,
\end{aligned}
$$

for arbitrary small $\varepsilon>0$. That means that we get for the unrefined mesh $(\mu=1)$ only an approximation order $\pi / \omega-\varepsilon\left(W^{1,2}(\Omega)\right.$-norm) or $2(\pi / \omega-\varepsilon)\left(L^{2}(\Omega)\right.$-norm). We conjecture that the $\varepsilon$ can be omitted. But this needs another way of proof, for example using the theory of interpolation spaces, compare [13] for the two-dimensional case. However, one can show by an example that these estimates cannot be improved further [1]. Numerical tests support the results, see $[4,8,10]$.

In the same way as above on can treat certain other boundary conditions. Conditions of third kind impose no further difficulties. Moreover, we can treat cases where Dirichlet boundary conditions are given only on a part of either $\Gamma_{\mathrm{B}}$ or $\Gamma_{\mathrm{M}}$. In particular, if the type of the boundary condition changes at the edge $M$ we have to substitute the expression $\pi / \omega$ by $\pi / 2 \omega$ in the whole text. Note further that for $\omega \geq \pi$ the solution is not any more contained in $W^{3 / 2+\varepsilon, 2}(\Omega)$ which implies that the interpolation operator $\mathrm{I}_{h}$ is not applicable to $u$.

However, if Dirichlet boundary conditions are given on (parts of) both $\Gamma_{\mathrm{B}}$ and $\Gamma_{\mathrm{M}}$ then neither $\mathrm{S}_{h} u \in V_{0 h}$ nor $\mathrm{E}_{h} u \in V_{0 h}$. In such cases we have to modify $\mathrm{S}_{h}$ or $\mathrm{E}_{h}$ near the Dirichlet boundary, as it was done by Clément for $\mathrm{C}_{h}$ [19]. But we will not develop this here.

\section{Summary}

The starting point of our investigation was the quasi-interpolation operator $\mathrm{Z}_{h}$ introduced by Scott and Zhang [30]. We have seen in Section 3 that anisotropic estimates of type $(m, \ell)$ are valid for $m=0$ but in general not for $m \geq 1$. Therefore we introduced three modifications and investigated the resulting operators $\mathrm{S}_{h}$, $\mathrm{L}_{h}$, and $\mathrm{E}_{h}$. In order to summarize and to compare the different Scott-Zhang type quasi-interpolation operators we give a tabular overview. For comparison we add also the results for the nodal interpolant $\mathrm{I}_{h}$ and for the operators $\mathrm{C}_{h}$ (Clément) and $\mathrm{O}_{h}$ (Oswald).

In Table 1 we find the element types which the operator is applicable for. Note the slight difference of tensor product type and tensor product elements in three dimensions. Tensor product type corresponds to transformation $(2.2,2.3)$, and tensor product means the restriction to transformation $(2.4)$. The operator $\mathrm{I}_{h}$ is widely investigated for more general elements including non-affine ones, see $[2,4,7]$. A comprehensive monograph is $[3]$.

Table 2 compares the conditions for which the stability estimate

$$
\left|\mathrm{Q}_{h} u ; W^{m, q}(e)\right| \lesssim(\operatorname{meas} e)^{1 / q-1 / p} \sum_{|\alpha| \leq \ell-m} h^{\alpha}\left|D^{\alpha} u ; W^{m, p}\left(S_{e}\right)\right|
$$

holds, $\mathrm{Q}_{h} \in\left\{\mathrm{C}_{h}, \mathrm{O}_{h}, \mathrm{Z}_{h}, \mathrm{~S}_{h}, \mathrm{~L}_{h}, \mathrm{E}_{h}, \mathrm{I}_{h}\right\}$. In the case of $\mathrm{S}_{h}$ and $\mathrm{E}_{h}$ we additionally proved stability in weighted Sobolev spaces. The estimate

$$
\left|\mathrm{Q}_{h} u ; W^{m, q}(e)\right| \leq(\operatorname{meas} e)^{1 / q-1 / p} h_{1}^{-\beta} \sum_{|\alpha|=m-1} \sum_{|t|=1} h^{t}\left\|D^{\alpha+t} u ; V_{\beta}^{1, p}\left(S_{e}\right)\right\|
$$

holds under the conditions given in Table 3. The approximation error estimate

$$
\left|u-\mathrm{Q}_{h} u ; W^{m, q}(e)\right| \lesssim(\text { meas } e)^{1 / q-1 / p} \sum_{|\alpha|=\ell-m} h^{\alpha}\left|D^{\alpha} u ; W^{m, p}\left(S_{e}\right)\right|
$$


TABLE 1. Treated finite elements.

\begin{tabular}{|c|c|c|}
\hline & $d=2$ & $d=3$ \\
\hline \multirow[t]{2}{*}{$\overline{\mathrm{Z}_{h}, \mathrm{C}_{h}, \mathrm{O}_{h}}$} & \multirow[t]{2}{*}{$\begin{array}{l}\text { tensor product meshes } \\
h_{1}, h_{2} \text { arbitrary }\end{array}$} & $\begin{array}{l}\text { meshes of tensor product type } \\
h_{1} \sim h_{2} \lesssim h_{3} \text { or } h_{1} \sim h_{2} \gtrsim h_{3}\end{array}$ \\
\hline & & $\begin{array}{l}\text { tensor product meshes } \\
h_{1}, h_{2}, h_{3} \text { independent }\end{array}$ \\
\hline $\mathrm{S}_{h}$ & $\begin{array}{l}\text { tensor product meshes } \\
h_{1} \lesssim h_{2}\end{array}$ & $\begin{array}{l}\text { meshes of tensor product type } \\
h_{1} \sim h_{2} \lesssim h_{3}\end{array}$ \\
\hline $\mathrm{L}_{h}$ & $\begin{array}{l}\text { tensor product meshes } \\
h_{1} \gtrsim h_{2}\end{array}$ & $\begin{array}{l}\text { meshes of tensor product type } \\
h_{1} \sim h_{2} \gtrsim h_{3}\end{array}$ \\
\hline \multirow[t]{2}{*}{$\overline{\mathrm{E}_{h}}$} & & $\begin{array}{l}\text { meshes of tensor product type } \\
h_{1} \sim h_{2} \lesssim h_{3}\end{array}$ \\
\hline & & $\begin{array}{l}\text { tensor product meshes } \\
h_{1} \lesssim h_{2} \lesssim h_{3}\end{array}$ \\
\hline \multirow[t]{3}{*}{$\mathrm{I}_{h}$} & \multirow[t]{2}{*}{$\begin{array}{l}\text { tensor product meshes } \\
h_{1}, h_{2} \text { arbitrary }\end{array}$} & $\begin{array}{l}\text { meshes of tensor product type } \\
h_{1} \sim h_{2} \lesssim h_{3} \text { or } h_{1} \sim h_{2} \gtrsim h_{3}\end{array}$ \\
\hline & & $\begin{array}{l}\text { tensor product meshes } \\
h_{1}, h_{2}, h_{3} \text { independent }\end{array}$ \\
\hline & $\begin{array}{l}\text { even for more general meshes, } \\
\text { see }[2-4,7]\end{array}$ & $\begin{array}{l}\text { even for more general meshes, } \\
\text { see }[2-4,7]\end{array}$ \\
\hline
\end{tabular}

TABLE 2. Conditions for the stability and error estimates.

\begin{tabular}{|l|l|}
\hline $\mathrm{C}_{h}, \mathrm{O}_{h}$ & $m=0,0 \leq \ell \leq k+1, p, q \in[1, \infty]$ \\
\hline $\mathrm{Z}_{h}$ & $m=0,1 \leq \ell \leq k+1, p, q \in[1, \infty]$ \\
\hline $\mathrm{S}_{h}$ & $0 \leq m \leq \ell-1,1 \leq \ell \leq k+1, p, q \in[1, \infty]$ \\
& for $m \geq 2$ triangles and tetrahedra are excluded \\
\hline $\mathrm{L}_{h}$ & $0 \leq m \leq \ell, 1 \leq \ell \leq k+1, p, q \in[1, \infty]$ \\
\hline $\mathrm{E}_{h}$ & $1 \leq m \leq \ell-1,1 \leq \ell \leq k+1, p, q \in[1, \infty]$ \\
\cline { 2 - 3 } & $m=0,2 \leq \ell \leq k+1, p, q \in[1, \infty]$ \\
\cline { 2 - 3 } & $m=0, \ell=1, p \in(2, \infty], q \in[1, \infty]$ \\
\hline $\mathrm{I}_{h}$ & $0 \leq m \leq \ell-1,1 \leq \ell \leq k+1, q=p$, \\
& $p>d / \ell$ if $\ell<d$ and $m=0$, \\
& $p>2$ if $d=3$ and $m=\ell-1>0$ \\
\cline { 2 - 2 } & $m=0, \ell=0, p=\infty, q \in[1, \infty]$ \\
\hline
\end{tabular}

TABle 3. Conditions for the stability in weighted Sobolev spaces.

\begin{tabular}{|l|l|}
\hline $\mathrm{C}_{h}, \mathrm{O}_{h}, \mathrm{Z}_{h}$ & not treated \\
\hline $\mathrm{S}_{h}$ & $0 \leq m \leq k, p, q \in[1, \infty], \beta<2-\frac{2}{p}, \beta \leq 1$ \\
& for $m \geq 2$ triangles and tetrahedra are excluded \\
\hline $\mathrm{L}_{h}$ & not treated \\
\hline $\mathrm{E}_{h}$ & $1 \leq m \leq k, p, q \in[1, \infty], \beta<2-\frac{2}{p}, \beta \leq 1$ \\
\cline { 2 - 2 } & $m=0, p \in(2, \infty], q \in[1, \infty], \beta<2-\frac{2}{p}, \beta \leq 1$ \\
\hline $\mathrm{I}_{h}$ & not treated in this form \\
\hline
\end{tabular}


TABLE 4. Restrictions in the applicability of the operators.

\begin{tabular}{|l|l|}
\hline $\mathrm{C}_{h}, \mathrm{O}_{h}, \mathrm{Z}_{h}$ & only $m=0$ \\
\hline $\mathrm{S}_{h}$ & $m=\ell$ excluded, only $m=0,1$ for simplices, in 3D only needle elements \\
\hline $\mathrm{L}_{h}$ & in 3D only flat elements \\
\hline $\mathrm{E}_{h}$ & $m=\ell$ excluded, restrictions on $p$ when $m=0, \ell=1$ \\
\hline $\mathrm{I}_{h}$ & $m=\ell$ excluded, restrictions on $p$ when $m=0, \ell<d$ or $m=\ell-1>0$ \\
\hline
\end{tabular}

holds if the conditions of Table 2 are satisfied and the parameters $\ell, p, m, q$ are such that the embedding $W^{\ell, p}(e) \hookrightarrow W^{m, q}(e)$ holds. The operator $\mathrm{I}_{h}$ plays an exceptional role also here, because estimate (8.2) is proved directly. The stability in the sense of (8.1) can be concluded via $\left|\mathrm{Q}_{h} u\right| \leq|u|+\left|u-\mathrm{Q}_{h} u\right|$. Second, we mentioned in Table 2 only $q=p$ (published result), but meanwhile the estimates are derived also for general $q \in[1, \infty]$ satisfying $W^{\ell, p}(e) \hookrightarrow W^{m, q}(e)$ [3]. Finally, anisotropic interpolation error estimates are derived in [9-11] for functions from weighted Sobolev spaces with $k=1, m=0,1, \ell=2, q=p$. For more general results we refer also to [3].

Some shortcomings of the operators are given in Table 4. Additionally, we state that Dirichlet boundary conditions $u=\left.g \in V_{h}\right|_{\Gamma_{1}}$ on $\Gamma_{1}$ can be satisfied on any part of $\partial \Omega$ for $\mathrm{Z}_{h}$ and $\mathrm{I}_{h}$, on parts of the boundary which are parallel to the $x_{1}$-axis $/ x_{1}, x_{2}$-plane for $\mathrm{S}_{h}$ and $\mathrm{L}_{h}$, and on parts of $\partial \Omega$ which are perpendicular to the $x_{1}, x_{2}$-plane for $\mathrm{E}_{h}$.

Finally, we mention that $\mathrm{S}_{h}$ and $\mathrm{E}_{h}$ have been successfully applied in the study of the Poisson problem in a domain with an edge where the singularity was treated with anisotropic mesh refinement, see Section 7. The operator $\mathrm{L}_{h}$ was applied by Becker [15] to show the stability and an approximation error estimate of the stabilized $Q_{1} / Q_{0}$-element pair in the context of the Stokes equation. $\mathrm{I}_{h}$ has been applied in the study of diffusion problems in domains with corners and edges [3,4,9-11,29], as well as for singularly perturbed convection-diffusion-reaction problems with anisotropic refinement in boundary layers $[2,3,6,7,20]$.

The work of the author is supported by DFG (German Research Foundation), Sonderforschungsbereich 393 . The author wishes also to thank an anonymous referee for many helpful comments.

\section{REFERENCES}

[1] Th. Apel, Finite-Elemente-Methoden über lokal verfeinerten Netzen für elliptische Probleme in Gebieten mit Kanten. Ph.D. thesis, TU Chemnitz (1991).

[2] Th. Apel, Anisotropic interpolation error estimates for isoparametric quadrilateral finite elements. Computing 60 (1998) $157-174$.

[3] Th. Apel, Anisotropic finite elements: Local estimates and applications. Teubner, Stuttgart (1999).

[4] Th. Apel and M. Dobrowolski, Anisotropic interpolation with applications to the finite element method. Computing 47 (1992) $277-293$.

[5] Th. Apel and B. Heinrich, Mesh refinement and windowing near edges for some elliptic problem. SIAM J. Numer. Anal. 31 (1994) 695-708.

[6] Th. Apel and G. Lube, Anisotropic mesh refinement in stabilized Galerkin methods. Numer. Math. 74 (1996) $261-282$.

[7] Th. Apel and G. Lube, Anisotropic mesh refinement for a singularly perturbed reaction diffusion model problem. Appl. Numer. Math. 26 (1998) 415-433.

[8] Th. Apel and F. Milde, Comparison of several mesh refinement strategies near edges. Comm. Numer. Methods Engrg. 12 (1996) 373-381.

[9] Th. Apel and S. Nicaise, Elliptic problems in domains with edges: anisotropic regularity and anisotropic finite element meshes, in Partial Differential Equations and Functional Analysis (In Memory of Pierre Grisvard), J. Cea, D. Chenais, G. Geymonat, and J.L. Lions Eds., Birkhäuser, Boston (1996) 18-34. Shortened version of Preprint SPC94_16, TU Chemnitz-Zwickau (1994).

[10] Th. Apel and S. Nicaise, The finite element method with anisotropic mesh grading for the Poisson problem in domains with edges. Technical report, TU Chemnitz-Zwickau (1996). Improved version of Preprint SPC94_16, TU Chemnitz-Zwickau (1994), available only via ftp, server ftp.tu-chemnitz.de, directory pub/Local/mathematik/Apel, file an1.ps.Z.

[11] Th. Apel and S. Nicaise, The finite element method with anisotropic mesh grading for elliptic problems in domains with corners and edges. Math. Methods Appl. Sci. 21 (1998) 519-549. 
[12] Th. Apel, A.-M. Sändig and J.R. Whiteman, Graded mesh refinement and error estimates for finite element solutions of elliptic boundary value problems in non-smooth domains. Math. Methods Appl. Sci. 19 (1996) 63-85.

[13] I. Babuška, R.B. Kellogg and J. Pitkäranta, Direct and inverse error estimates for finite elements with mesh refinements. Numer. Math. 33 (1979) 447-471.

[14] A.E. Beagles and J.R. Whiteman, Finite element treatment of boundary singularities by augmentation with non-exact singular functions. Numer. Methods Partial Differential Equations 2 (1986) 113-121.

[15] R. Becker, An adaptive finite element method for the incompressible Navier-Stokes equations on time-dependent domains. Ph.D. thesis, Ruprecht-Karls-Universität Heidelberg (1995).

[16] J.H. Bramble and S.R. Hilbert, Estimation of linear functionals on Sobolev spaces with applications to Fourier transforms and spline interpolation. SIAM J. Numer. Anal. 7 (1970) 112-124.

[17] J.H. Bramble and S.R. Hilbert, Bounds for a class of linear functionals with applications to Hermite interpolation. Numer. Math. 16 (1971) 362-369.

[18] P.G. Ciarlet, The finite element method for elliptic problems. North-Holland, Amsterdam (1978).

[19] P. Clément, Approximation by finite element functions using local regularization. RAIRO Anal. Numér. 9 (1975) $77-84$.

[20] M. Dobrowolski and H.-G. Roos, A priori estimates for the solution of convection-diffusion problems and interpolation on Shishkin meshes. Z. Anal. Anwendungen 16 (1997) 1001-1012.

[21] T. Dupont and R. Scott, Polynomial approximation of functions in Sobolev spaces. Math. Comput. 34 (1980) $441-463$.

[22] R.G. Durán, Error estimates for 3-d narrow finite elements. Math. Comput. 68 (1999) 187-199.

[23] A. Kufner and A.-M. Sändig, Some Applications of Weighted Sobolev Spaces. Teubner, Leipzig (1987).

[24] G. Kunert, Error estimation for anisotropic tetrahedral and triangular finite element meshes. Preprint SFB393/97-17, TU Chemnitz (1997).

[25] M.S. Lubuma and S. Nicaise, Dirichlet problems in polyhedral domains II: approximation by FEM and BEM. J. Comput. Appl. Math. 61 (1995) 13-27.

[26] M.S. Lubuma and S. Nicaise, Finite element method for elliptic problems with edge corners. J. Comput. Appl. Math. (submitted).

[27] L.A. Oganesyan and L.A. Rukhovets, Variational-difference methods for the solution of elliptic equations. Izd. Akad. Nauk Armyanskoi SSR, Jerevan (1979). In Russian.

[28] P. Oswald, Multilevel Finite Element Approximation: Theory and Applications. Teubner, Stuttgart (1994).

[29] T. von Petersdorff, Randwertprobleme der Elastizitätstheorie für Polyeder — Singularitäten und Approximationen mit Randelementmethoden. Ph.D. thesis, TH Darmstadt (1989).

[30] L.R. Scott and S. Zhang, Finite element interpolation of non-smooth functions satisfying boundary conditions. Math. Comp. 54 (1990) 483-493.

[31] N.A. Shenk, Uniform error estimates for certain narrow Lagrangian finite elements. Math. Comp. 63 (1994) $105-119$.

[32] K. Siebert, An a posteriori error estimator for anisotropic refinement. Numer. Math. 73 (1996) 373-398.

[33] E.P. Stephan and J.R. Whiteman, Singularities of the Laplacian at corners and edges of three-dimensional domains and their treatment with finite element methods. Math. Methods Appl. Sci. 10 (1988) 339-350.

[34] H. Walden and R.B. Kellogg, Numerical determination of the fundamental eigenvalue for the Laplace operator on a spherical domain. J. Engrg. Math. 11 (1977) 299-318. 